\title{
Les groupes bulbes Projets et perspectives
}

\author{
par S. Casacci \\ Directeur Technique, Neyrpic-Creusot-Loire \\ (N-CL.)
}

\begin{abstract}
Ont participé plus particulièrement à l'élaboration de ce document: M. LE PLOMB (C.N.R.) - MM. ANDRÉ, CAVAILLÉ, FRANÇOIS (E.D.F.) MM. BOSC, SAURON (Neyrpic) - MM. MOULIN, MÉGNINT, $\mathrm{M}^{\mathrm{m}+\mathrm{C}}$ CARRICHON et $\mathrm{M}^{1+\mathrm{le}}$ GAUD (Neyrpic-Cl.)
\end{abstract}

L'équipement de la centrale marémotrice de la Rance et celui des grands fleuves français, à l'aide de groupes bulbes, constitue une réussite dans la recherche de l'économie des installations de basses chutes. Il y a plus de vingt ans, Electricité de France lançait des concours d'idées pour la réduction des coûts des centrales de basses chutes et envisageait d'équiper des microcentrales. A cette époque, les études sur l'aménagement des centrales marémotrices se poursuivaient et incitaient ingénieurs-projeteurs et constructeurs à rechercher des types de turbo-machines pouvant fonctionner en turbine et en pompe, pour les deux sens d'écoulement, tout en comportant des conduits hydrauliques de formes simples et, partant, économiques.

Ces recherches, pour l'amélioration de la rentabilité des microcentrales et des centrales marémotrices, conduisirent à la mise au point des groupes «bulbes-amont». Le choix de ces machines axiales s'imposait; les conduits hydrauliques, pratiquement axisymétriques, permettaient d'envisager u? accroissement des puissances spécifiques et conduisaient à une réduction sensible du coût du génie civil, à cause de leurs formes simples et de leurs dimensions réduites par; rapport à celles des Kaplan verticales de même productivité.

Ces machines ne virent pas le jour sans peine. Avec la première génération de turbines, du type Castet, dont lo diamètre de la roue restait inférieur à deux mètres, un pas décisif dans la connaissance de nombreux problèmes hydrau. liques et mécaniques fût franchi. Le passage à la seconde génération de machines, à double réglage, dont les roues dépassaient quatre mètres de diamètre s'effectua avec précaution. Les efforts hydrauliques, appliqués à la roue en porte-à-faux au cours des régimes transitoires, étaient ma connus; la détermination sur modèle de ces efforts, en régime permanent ou transitoire, présente de très grandes difficultés; pour des investissements élevés, ce type d'essais sur modèle risquait de ne fournir que des résultats incomplets. Nous avons préféré progresser prudemment dans l'accroissement des puissances unitaires. A chaque pas franchi, des essais, sur machines industrielles et sur modèles réduits, nous permettaient d'aborder avec grande sûreté une nouvelle extrapolation. La conception des alternateurs dans l'air atmosphérique ou comprimé posait beaucoup plus de problèmes que celle des alternateurs de la première génération qui, eux, comportaient des rotors baignant dans l'huile.

Le complet succès de la mise en route du groupe expérimental de Saint-Malo, en 1959, permettait d'envisager avec confiance l'équipement de l'usine marémotrice de la Rance et des grands fleuves français (Rhône, Rhin, Garonne).

Aujourd'hui, les 212 groupes bulbes construits constituent la récompense de nos efforts. Nous allons analyser, dans cette étude, les principales étapes du développement des machines axiales. Nous nous attacherons surtout à l'étude des problèmes techniques spécifiques. Nous insisterons plus particulièrement sur les groupes bulbes à double réglage comportant des roues de grand diamètre. Le lecteur trouvera, dans le rapport de $M$. Cotillon, une vue synoptique du développement des groupes bulbes et, dans celui de M. Beslin, un exemple d'application à l'équipement de l'installation de Caderousse. Cette installation, comme celle d'Avignon, comporte des groupes bulbes à double réglage et des machines à distributeur et pales fixes; ces groupes PF-DF constituent la troisième génération.

Nous analyserons enfin les problèmes que soulève l'accroissement des puissances unitaires des groupes axiaux du type «bulbe-amont». 


\section{1. - Tracé hydraulique des groupes bulbes}

Au début de nos recherches sur les groupes bulbes, nous cherchions à augmenter la vitesse de la machine et à réduire le diamètre de la roue, tout en conservant de bons rendements. Nous cherchions donc à accroître les puissances spécifiques par augmentation de la vitesse et de la puissance.

Nous poursuivions à l'époque, depuis de nombreuses années, des études sur les écoulements dans les turbines Kaplan [1]. Nos essais sur les distributions de vitesse, à l'entrée et à la sortie des roues, montraient que des pertes importantes se produisaient dans l'amenée et dans l'aspirateur, dès que les puissances spécifiques devenaient assez élevées. Les conduits hydrauliques des groupes bulbes, quasi axisymétriques, devaient nécessairement avoir des pertes relativement moins importantes que celles mesurées dans les turbines Kaplan. Les meilleures caractéristiques des conduits hydrauliques des groupes axiaux devaient donc rendre possible l'utilisation de puissances spécifiques plus élevées pour une chute donnée. Nos recherches nous ont, effectivement, permis de mettre au point des machines axiales présentant ces qualités. Pour la chute de dimensionnement de l'installation, à rendement égal, le groupe bulbe a un diamètre de roue inférieur à celui de la Kaplan de même puissance; de plus, pour des chutes plus faibles que la chute caractéristique, les puissances fournies par la turbine axiale restent supérieures à celles développées par la Kaplan.

\subsection{Aspirateur.}

L'énergie cinétique, à l'aval immédiat de la roue, atteint une valeur voisine de l'énergie totale de la chute, ce qui montre l'importance de l'aspirateur dans les machines de grandes puissances spécifiques. Le bon fonctionnement du diffuseur constitue donc l'élément essentiel de la réussite des groupes axiaux et mérite qu'on précise les problèmes posés par le tracé des aspirateurs.

Un écoulement axial uniforme à la sortie de la roue, d'ailleurs difficile à obtenir même pour un seul point de fonctionnement, donnerait un excellent rendement, à condition de choisir un demi-angle au sommet assez faible $\alpha_{0}$. Pour cet $\alpha_{o}$, cependant, les longueurs des aspirateurs atteindraient des valeurs économiquement inacceptables et l'ingénieur hydraulicien se voit, comme toujours, obligé à un compromis qui l'amène à choisir une section de sortie égale à quatre fois la section de la roue environ et une longueur raisonnable; le demi-angle, au sommet $\alpha$ de cet aspirateur, dépasse alors la valeur $\alpha_{\theta}$ ce qui entraine des risques de décollements sur la paroi extérieure. Pour éviter ces décollements, il faut laisser à l'écoulement, à l'entrée de la roue, un certain moment cinétique par rapport à l'axe de la machine. Les choix de ce moment cinétique résiduel et de la loi des répartitions des vitesses tangentielles le long du rayon restent choses délicates, car les pertes dans l'aspirateur ne proviennent pas uniquement des décollements à la paroi, mais aussi des courants de retour dans la partie centrale. Lorsque le moment cinétique imprimé à l'écoulement, à l'entrée de l'aspirateur, devient trop important, les pertes par recirculation croissent très rapidement.

Les graphiques 1.1 et 1.2 donnent, respectivement, l'allure des pertes dans l'aspirateur d'un groupe bulbe et d'une Kaplan. On a porté, en ordonnées, les pertes et, en abscisses,

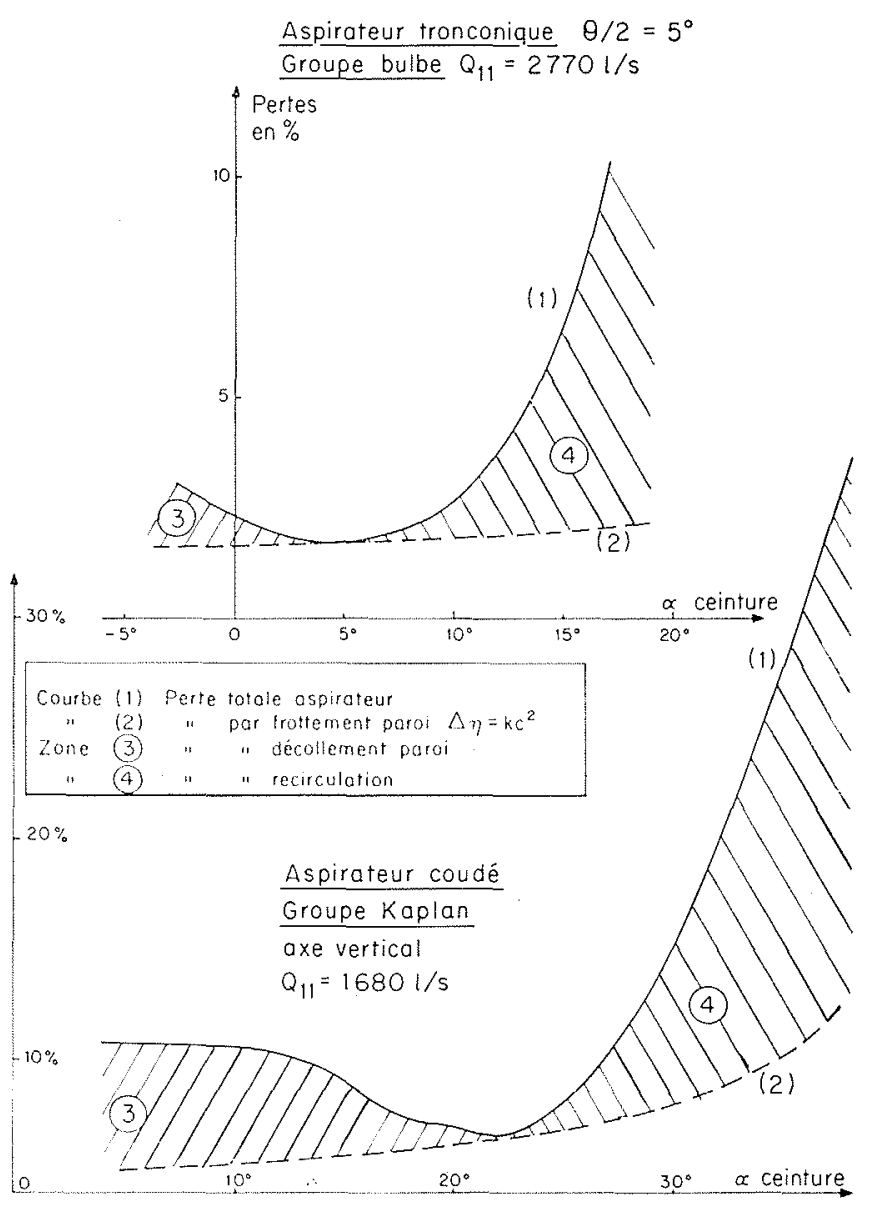

1.1 et 1.2/ Allure des pertes des aspirateurs.

l'angle que fait la vitesse absolue avec l'axe de la machine, cette vitesse de référence concerne l'entrée de l'aspirateur, côté ceinture. Les pertes minimales correspondent à un angle assez bien défini, donc à un moment cinétique donné. On remarque que les pertes croissent plus vite pour les valeurs supérieures à l'angle optimal que pour les valeurs inférieures. En fait, l'écoulement dans l'aspirateur, très complexe, dépend du tracé de l'ensemble des conduits hydrauliques et de celui de la roue.

Nous conclurons en précisant que les pertes dans les aspirateurs tronconiques droits proviennent, presque uniquement, des décollements, des turbulences et des gros tourbillons qui désorganisent l'écoulement; les pertes, par frottement sur la paroi, restent faibles. L'énergie cinétique, à la sortie de l'aspirateur d'un groupe bulbe, correspond à 1,4 à 1,5 fois le $\mathrm{V}_{m}^{2} / 2 \mathrm{~g}$ moyen, cette énergie peut atteindre $3 \mathrm{~V}^{2}{ }_{m} / 2 g$ pour un aspirateur de Kaplan. La récupération partielle de cette énergie, à l'aval de l'aspirateur, s'effectue dans de meilleures conditions pour les groupes bulbes que pour les Kaplan, car le champ des vitesses, à la sortie de laspirateur, est beaucoup plus homogène pour les premières machines que pour les secondes. 


\subsection{Conduits d'amenée.}

Au point de dimensionnement de l'installation, à rendement égal, le diamètre de la roue du groupe bulbe est inférieur à celui de la Kaplan de même puissance. Les pertes, dans l'amenée des deux machines, restent comparables, bien que les dimensions des conduits amont et du distributeur du groupe bulbe soient plus faibles que celles de la Kaplan. Nous ne recherchions pas une amélioration des rendements, mais une amélioration de l'économie d'ensemble des basses chutes. Nous préférions donc, à pertes égales, réduire les dimensions du distributeur, organe qui représente une part importante du prix de la turbine. Un encombrement limité des conduits amont permettrait, de plus, de diminuer la longueur de la centrale et de loger l'ensemble du groupe entre deux joints plans verticaux et parallèles. On obtenait ainsi une plus grande puissance équipée pour une longueur de centrale donnée [2], [3]. Ces dispositions d'ensemble exigeaient que le diamètre du bulbe, et donc du stator de l'alternateur, restât inférieur au diamètre de la roue. Le développement de ces machines se trouvait donc conditionné par la possibilité de construire des alternateurs de diamètre réduit. Cette condition était encore plus impérative pour les groupes marémoteurs qui devaient fonctionner en double flux, le conduit amont servant de diffuseur pour le fonctionnement en turbine inversée et en pompe directe.

Nous allons examiner succinctement les difficultés que pose le tracé hydraulique de l'amenée, pour un rapport $\varnothing_{\text {ilt }} / \varnothing_{\text {roue }}$ voisin de $0,8 / 0,9$. Les problèmes à résoudre consistent principalement :

- à réduire les pertes singulières, sillages, décollements provoqués par les appuis, la forme des conduits, etc.

- à obtenir une répartition correcte de vitesse à l'entrée de la roue, quil rende possible un tracé de grille d'aubes tournantes à bon rendement et à grande puissance spécifique.

L'étude des formes hydrauliques des conduits amont se trouve fort compliquée par la présence des appuis du groupe, elle est plus aisée pour les dispositions en chambre d'eau. L'étude comparative des dispositions, en conduite et en chambre d'eau, devrait être reprise en examinant les aspects hydrauliques, les dispositions mécaniques d'ensemble et leur influence sur les ouvrages de génie civil. Signalons que la définition des formes d'entonnement et des pertes dans les grilles reste un problème d'optimisation délicat. Pour les groupes de la Rance, le conduit amont, servant aussi de diffuseur pour la turbine inversée et pour la pompe directe, l'obstruction des supports a été réduite au strict minimum.

Dans le cas des bulbes de rivière, le dimensionnement de l'entonnement, l'obstruction des appuis et le dimensionnement réduit du distributeur expliquent la majeure partie des pertes dans le conduit amont. Dans la conception actuelle, on ne peut envisager de supporter le groupe au droit de l'alternateur à cause du manque de rigidité de la carcasse. L'accroissement des puissances unitaires, s'il s'avère utile, conduirait à des groupes comportant des roues de 7,5 à $8 \mathrm{~m}$ de diamètre. On ne pourrait plus adopter une conception en une seule pièce pour les carcasses de ces groupes. Ces carcasses, en plusieurs parties, seraient nécessairement beaucoup plus rigides et il deviendrait possible de supporter le groupe au droit de l'alternateur. Cette disposition permettrait de réduire, dans les zones à vitesse d’écoulement éle- vée, l'obstruction des apppuis et, par conséquent, les pertes dans le conduit amont.

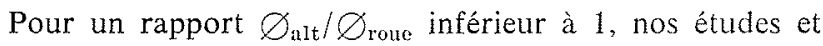
nos essais nous ont conduits à l'adoption d'un distributeur dans lequel les axes des directrices font un angle de $65^{\circ}$ avec l'axe du groupe. Les formes des parois intérieure et extérieure du distributeur et 'de l'avant-distributeur ont demandé de longues mises au point sur modèle pour obtenir un écoulement sans décollement et une répartition correcte des vitesses à l'entrée de la roue. Tout d'abord, une bonne alimentation au droit du moyeu constitue l'une des conditions indispensables pour atteindre de bonnes performances; en effet, les profils de la roue, au voisinage du moyeu, travaillent en général dans de meilleures conditions que ceux proches de la ceinture et un décollement sur la paroi intérieure du distributeur compromettrait très sensiblement les rendements 'de la roue.

La répartition de l'angle d'injection, le long du rayon à l'entrée de la roue, con'ditionne aussi le rendement de cette dernière; cet angle doit croître du moyeu à la ceinture. Pour ne pas augmenter le prix des distributeurs mobiles, nous avons toujours envisagé des formes simples de directrices; les génératrices des profils des aubes concourent au sommet du cone contenant les axes des directrices, ce sommet constitue donc un centre d'homothétie pour les différents profils. Ces formes simplifient considérablement la fabrication, la définition géométrique et l'usinage du distributeur. Le tracé optimal de la roue exigerait que les directrices possèdent une certaine torsion; cette torsion ne pourrait être obtenue que par une déformation des profils au-delà de la génératrice de contact. Ces tracés de directrices conduiraient à un allongement des profils, une augmentation des efforts de manœuvre et correspondraient donc à un renchérissement du 'distributeur économiquement inacceptable.

Nos recherches, sur les groupes à distributeur mobile, nous ont permis d'obtenir une répartition correcte des vitesses à l'entrée de la roue, en jouant sur la forme des parois, sur la géométrie du distributeur et sur la forme des profils homothétiques constituant les directrices.

L'accroissement des puissances unitaires des groupes bulbes, s'il s'avère économiquement intéressant pour l'économie d'ensemble des installations, exigera de nouvelles études hydrauliques. Jusqu'à présent, pour les groupes du type «bulbe-amont » à un seul appui (Saint-Malo, Rance) ou à deux appuis (groupes Rhône, Rhin, etc.), le rapport

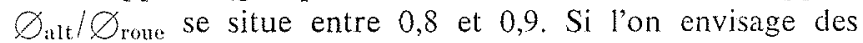
groupes de 7,5 à $8 \mathrm{~m}$ de diamètre de roue, ce rapport devra être augmenté jusqu'à $1,2-1,3$ pour faciliter la conception des carcasses d'alternateur en plusieurs pièces; rappelons que les diamètres de carcasses des groupes construits jusqu'ici ne dépassent pas $5,600 \mathrm{~m}$ et permettent une conception en une seule pièce. L'augmentation du rapport $\varnothing_{\text {ult }} / \varnothing_{\text {roue }}$ conduit à modifier le tracé hy'draulique de l'amenée et du distributeur. Pour ne pas trop allonger le groupe, il faut diminuer le demi-angle au sommet du distributeur conique, ce qui entraîne un accroissement des courbures de l'écoulement à l'entrée du distributeur. Il semble possible, en première analyse, de concevoir des groupes bulbes de puissances spécifiques élevées, avec des rapports $\varnothing_{\text {alt }} / \varnothing_{\text {rank }}$ voisins de 1,2 à 1,3, en adoptant un demi-angle au sommet du distributeur de l'ordre de 40 à $50^{\circ}$. L'adoption de cet angle permettrait de ne pas trop accroître le diamètre extérieur du distributeur, mais poserait, à nouveau, le problème de l'alimentation de la roue. Ce tracé laisserait plus de place 
à l'intérieur du bulbe et rendrait peut-être possible une commande intérieure du vannage. L'augmentation du rapport $\varnothing_{\text {alt }} / \varnothing_{\text {ronte }}$ conduirait à un léger accroissement de l'entre-axe des groupes, mais la réduction des pertes dans les grilles viendrait compenser cet inconvénient; ces pertes sont en général sous-estimées.

Les groupes simplifiés à distributeur fixe et pales mobiles (DF-PM) ou à distributeur fixe et pales fixes (DF-PF) ont déjà trouvé des applications, dont les plus spectaculaires concernent les installations de Sauveterre ot de Caderousse, toutes deux sur le Rhône. Des machines (DF-PF) équiperont ces deux centrales; leur distributeur fixe comporte des aubes maillées pour assurer la répartition recherchée des vitesses à l'entrée de la roue. Les directrices creuses, prévues pour l'accès à l'intérieur du bulbe ou pour le passage des tuyauteries des câbles, etc., compliquent beaucoup le tracé et la construction; le déplacement des accès vers l'amont rend la conception hydraulique et mécanique plus aisée.

\subsection{Roue.}

Nous n'avons pas ici pour dessein d'analyser les difficultés rencontrées dans l'étude des tracés des roues hélices à pales mobiles. Nous ferons, seulement, quelques remarques sur les problèmes posés par les machines de vitesses spécifiques élevées. La réussite de telles recherches nécessite des allersretours de l'étude théorique aux essais sur modèle.

Les conditions optimales à l'entrée seraient relativement simples à définir, encore qu'il ne soit pas aisé de concevoir une amenée et un distributeur économiques qui donnent le champ de vitesse recherché, d'autant plus que les écoulements, même dans les groupes bulbes, ne sont pas strictement axisymétriques.

Les conditions à la sortie de la roue, doivent être optimales pour la roue et pour l'aspirateur; en fait, on sacrifie un peu les performances de la roue pour obtenir un bon rendement d'aspirateur, rendement qui, comme nous l'avons précisé ci-avant, est un paramètre fondamental.

Les conditions d'entrée et de sortie définies, on détermine des turbines élémentaires limitées par des surfaces de révolution (pas nécessairement cylindriques). Ces machines élémentaires sont données par un programme de calcul.

La répartition des pressions et des vitesses, le long des aubes de ces turbines élémentaires, est calculée en supposant le fluide parfait et l'écoulement irrotationnel. Une correction se fait, ensuite, en introduisant l'effet de la couche limite. Une transformation conforme permet de passer de la grille d'aubes, tracée sur une surface de révolution, à une grille d'aubes planes. Les répartitions des vitesses et des pressions calculées permettent de vérifier que le choix initial des turbines élémentaires satisfait à certaines conditions : l'équilibre radial par exemple. On procède par iteration pour obtenir les turbines élémentaires définitives. Cette méthode constitue un modèle mathématique quasi-tridimensionnel [4], [5], [6], qui permet de prendre en compte l'effet des vitesses radiales; elle n'épuise cependant pas les problèmes.

Les investissements nécessaires, pour contrôler l'efficacité des méthodes théoriques de tracés, atteignent rapidement des montants importants dès que l'on envisage des recherches assez fines [7]. Les résultats des essais se révèlent, d'ailleurs, d'une interprétation délicate pour les mesures sur les aubes tournantes et, même, pour les écoulements à l'entrée et à la sortie de la roue. Toutefois, des vérifications partielles peuvent être effectuées avec des investissements économiquement acceptables, comme, par exemple : amélioration des caractéristiques, définition des zones les plus sensibles à la cavitation, visualisation des écoulements dans la roue, etc.

\subsection{Cavitation.}

Les turbines qui équipent des installations de basses chutes fonctionnent souvent sous des chutes très variables. Ces marnages relatifs importants rendent plus délicate la définition du calage des machines susceptibles d'assurer une exploitation acceptable. Les groupes bulbes entrent dans la catégorie des turbines alimentées par des chutes fortement variables et méritent, donc, que les conditions de cavitation soient analysées de façon approfondie, d'autant plus que ces machines présentent certains aspects particuliers du fait de leur disposition à axe horizontal et, surtout, à cause de leur puissance spécifique élevée.

Rappelons que la méthode d'essais consiste, pour un point de fonctionnement donné, à observer, pour des $\sigma$ décroissants, l'apparition de la cavitation en tout point de la pale. En plus des $\sigma$ de début de cavitation $\sigma_{d}$, on note tous les phénomènes susceptibles de renseigner sur les conditions de cavitation: formes des zones soumises à des cavitations, leur développement avec la réduction du $\sigma$, stabilité des écoulements, bruit, etc. Pour des raisons économiques évidentes, on ne peut adopter un calage qui garantirait la machine contre toute cavitation, les règles de l'art consistent à choisir une implantation qui assure une exploitation acceptable.

Maîtres d'œuvres et constructeurs utilisent en général, pour définir le calage d'une machine, le $\sigma$ standard $\sigma_{s}$ qui, pour un point de fonctionnement donné, caractérise le $\sigma$ au-dessous duquel les performances de la machine se détériorent plus ou moins rapidement; le $\sigma_{s}$ se mesure assez aisément, mais il caractérise une cavitation plus ou moins généralisée. Presque toujours, quel que soit le point de fonctionnement, lorsqu'on approche du $\sigma_{s}$, une grappe de cavitation importante se produit au voisinage du moyeu; ce n'est en général que lorsque, en réduisant le $\sigma$, cette grappe dépasse le bord de sortie de l'aube, que les performances de la machine commencent à se détériorer. Mais les écoulements dans la roue peuvent être fort différents avant d'atteindre le $\sigma_{s}$, suivant les points de fonctionnement considérés.

Les observations sur l'apparition et le développement de la cavitation constituent donc un ensemble de renseignements précieux, sans lequel les courbes des $\sigma_{s}$, tracées sur la colline, n'auraient que peu de signification. Ces observations peuvent, quelquefois, amener les constructeurs à modifier le tracé des pales, mais elles servent surtout à définir, pour les différentes zones de fonctionnement, les marges à adopter. Après une période d'exploitation de durée suffisante, l'analyse des usures, même faibles, sur la machine industrielle, à la lumière des observations faites sur modèle, permet de définir les types de cavitation qui provoquent des usures et de contrôler que les marges adoptées restent suffisantes. Ces allers-retours, du modèle à la machine industrielle, constituent une démarche très fructueuse pour la connaissance des phénomènes de cavitation et de leurs conséquences. 
On pourrait se baser sur les $\sigma_{d}$ caractérisant le début de cavitation pour définir le calage, ce ne serait pas chose facile. Les $\sigma_{l}$ ne se rapportent pas à la même zone de l'aubage suivant les points de fonctionnement et, de plus, ils peuvent concerner des types de cavitation très différents. Coter la colline en $\sigma$ admissibles, partant des $\sigma_{d l}$, exigerait de connaître les types de cavitation dangereuse et l'intensité acceptable, compte tenu des conditions de fonctionnement de la machine industrielle. Remarquons que le $\sigma_{d}$ peut se transposer assez correctement du modèle au prototype mais que, par contre, l'étendue de la poche de cavitation dépend de la chute. La référence au $\sigma_{s}$, malgré ses imperfections, semble donc utile, surtout si l'on a'dopte des marges différentes suivant les zones de fonctionnement. Ces marges doivent tenir compte de toutes les influences secondaires: tolérance de fabrication des profils [8], majoration des rendements, types de cavitation, etc.

La définition du calage, pour les turbines Kaplan verticales, s'effectue à partir des $\sigma_{s}$ déterminés sur mơdèle. Le choix du $\sigma$ de l'installation $\sigma_{i}$ dépen'd des $\sigma_{s}$ caractérisant le tracé de la machine, du matériau utilisé, des constatations faites sur les machines industrielles, des observations relevées sur modèle, etc. Ce choix se traduit par la définition d'un $\sigma_{i} / \sigma_{s}$ à préciser sur la colline de la turbine. Nous avons adopté les mêmes règles pour la détermination du calage des groupes bulbes et penson's, qu'en a'doptant les mêmes rapports $\sigma_{i} / \sigma_{s}$ définis à partir des résultats d'exploitation des turbines Kaplan, nous obtenons une marge de sécurité suffisante, d'autant plus que toutes les observations sur l'apparition et le développement de la cavitation ont été faites au point haut de la roue. Le bon fonctionnement des nombreux groupes bulbes en service depuis de longues années, vient confirmer ces critères de choix et prouve que la variation de la pression statique sur la pale, pendant un tour de roue, ne semble pas être la source de phénomènes particuliers; nous continuons cependant l'analyse de cet aspect du problème.

Nous allons analyser succinctement les problèmes de cavitation spécifiques aux groupes bulbes, liés à la puissance spécifique élevée et aux marnages relatifs importants. Il va sans dire que, pour une chute de dimensionnement donnée, l'accroissement de la puissance spécifique entraine une augmentation des $\sigma_{s}$ et, par conséquent, exige un enfoncement plus important. Le tracé des pales capables de développer de gran'des puissances spécifiques, comporte des profils relativement minces vers la périphérie. On peut constater, sur des pales en acier ordinaire, des usures à l'entrée côté extrados ou intrados car ces types de profils sont plus sensibles à la cavitation. Rappelons que, sur une colline graduée, par exemple en $\mathrm{Q}_{11}, n_{11}$, on peut définir une ligne $\mathrm{Q}_{11}=\mathrm{Q}_{11}\left(n_{11}\right)$ qui correspond à un angle d'incidence optimal des aubes. A droite d'une bande centrée sur cette courbe, zone qui correspond aux faibles chutes, les incidences deviennent négatives et des cavitations peuvent apparaître à l'entrée vers la périphérie côté intrados; à gauche de cette bande, c'est-à-dire pour les fortes chutes, les incidences deviennent positives et des cavitations risquent de se produire côté extrados à l'entrée et vers la ceinture. Précisons que pour ces tracés de grandes puissances spécifiques, des écarts d'incidences relativement faibles, différents d'ailleurs à la ceinture et au moyeu, peuvent provoquer des cavitations à l'entrée. Remarquons que ces cavitations à l'entrée, tant sur l'extrados que sur l'intrados, restent peu influencées par le $\sigma$. Les roues en acier ordinaire sur les- quelles ont été constatées des usures à l'entrée, provoquées par ces types de cavitation, se comportent bien après un rechargement en acier inoxydable des zones érodées.

Les chutes et les débits fortement variables, qui caractérisent la marche des groupes bulbes, exigent une analyse approfondie des conditions de cavitation pour toutes les zones d'utilisation de ces machines. Le mode de travail de la grille tournante varie beaucoup suivant les points de fonctionnement sur la colline. Nous avons déjà signalé que la chute des performances correspond, presque toujours, à l'apparition d'une grosse poche de cavitation, au droit du moyeu, qui s'étend au-delà de l'arête de sortie des pales. Les usures dangereuses ne se produisent cependant pas dans la zone du moyeu, ce qui montre que chute des caractéristiques et usures ne sont pas nécessairement liées. On trouve peu de différence sur la forme des courbes donnant le rendement $\eta=\eta(\sigma)$, au-dessous du $\sigma_{s}$ la chute du $\eta$ ne semble pas caractériser les points de fonctionnement (voir fig. 1.3). Pour définir les $\sigma$ d'utilisation, il faut analyser, sur modèle, l'écoulement pour des $\sigma$ variant du $\sigma_{d}$ au $\sigma_{s}$. Ainsi, pour les forts débits à faibles chutes, la cavitation de profil vers la sortie à l'extraidos, côté ceinture, se manifeste avant d'atteindre le $\sigma_{s}$ (fig. 1.4). L'intensité de ce type de cavitation augmente depuis le $\sigma_{d}$ jusqu'au $\sigma_{s}$; pour ces zones de fonctionnement, il faudra donc choisir un $\sigma_{u}=\sigma_{i}$ caractéristique de l'usure, tel que $\sigma_{i} / \sigma_{s}$ soit suffisant pour se garantir contre les usures anormales. Le rapport $\sigma_{u} / \sigma_{s}$ croit avec le débit spécifique. Les marches aux faibles débits et hautes chutes ne présentent, en général, pas de cavitation de profil pour des $\sigma>\sigma_{s}$ (voir fig. 1.5).

Nous dirons enfin quelques mots sur les usures des ceintures qui peuvent être provoquées par des cavitations sur les pales. Signalons, qu'en ce qui concerne les cavitations marginales, les bulbes ne posent pas de problème spécifique. Des cavitations à l'entrée des pales, côté périphérie, peuvent provoquer des usures de la ceinture dans le plan moyen de la roue. Pour se garantir contre ces usures, qui sont de faible intensité, on peut adopter des ceintures en acier inoxydable ou prévoir des rechargements, surtout pour la

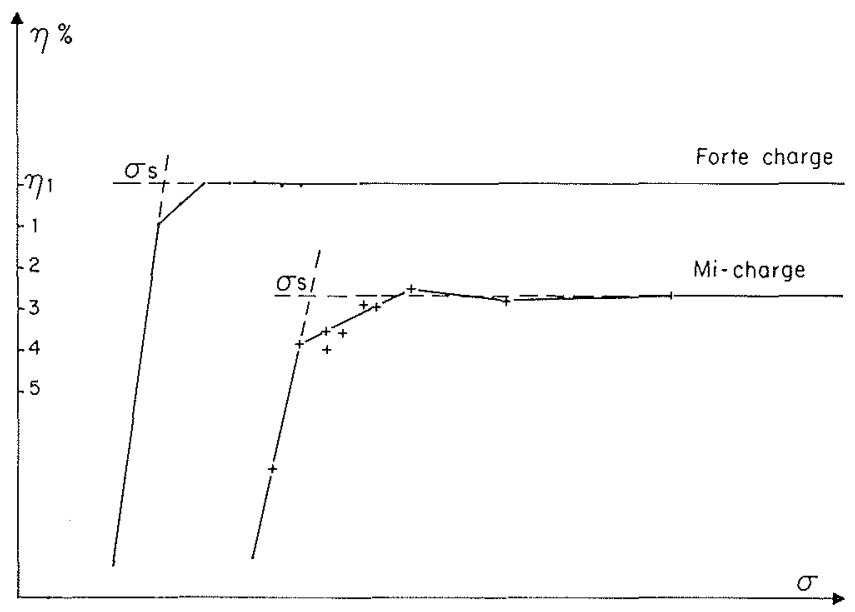

1.3/ Forme de la courbe de chute de rendement en deça du $\sigma_{s}$ pour différentes charges. 
partie supérieure. Des usures peuvent aussi se produire sur la ceinture au-delà du col, elles ont pour cause les cavitations qui prennent naissance sur les aubes à la sortie, côté extrados; un enfoncement suffisant évite alors toute usure anormale.

La figure 1.6 représente une colline de groupe bulbe sur laquelle sont mentionnés les principaux renseignements permettant de définir le calage.

Nous terminerons par quelques remarques sur les difficultés que pose la définition des lois de conjugaison des deux organes de réglage. Disons, tout d'abord, que si les dispositifs de commande actuels permettent d'obtenir, avec une précision bien suffisante, les lois de conjugaison prévues, la difficulté vient du choix de ces lois. Les lois de conjugaison adoptées sont celles définies par les essais sur modèle, car les mesures sur machines industrielles dont on dispose, ne permettent pas de définir des méthodes de correction suffisamment sûres. Les facteurs susceptibles de modifier ces lois de conjugaison sont connus, mais leur influence peut difficilement être chiffrée: majorations des rendements et des puissances, définition des chutes nettes sur modèle et sur machines industrielles, etc. Les essais sur modèle montrent, qu'en général, l'influence d'une faible déconjugaison reste peu sensible sur les phénomènes de cavitation; il est donc raisonnable d'adopter des lois de conjugaison définies sur modèle si l'on ne dispose pas d'une méthode de correction permettant de passer du modèle au prototype.

\subsection{Puissances spécifiques des groupes bulbes.}

Nous avons fait, pour des installations existantes, une étude statistique des limites d'utilisation maximales des tur- bines Kaplan et des groupes bulbes, compte tenu du marnage caractérisant chacune des usines. Le graphique 1.7 donne ces limites d'utilisation dans le système de coordonnées $\mathrm{Q}_{11}, n_{11}$. L'examen de ces données statistiques montre que le débit spécifique $Q_{11}$ maximal des machines axiales atteint $4000 \mathrm{l} / \mathrm{s}$, tandis que celui des Kaplan dépasse rarement $2600 \mathrm{l} / \mathrm{s}$. Les $n_{11}$ des groupes bulbes arrivent à des valeurs de $250 \mathrm{tr} / \mathrm{mn}$, ceux des Kaplan vont jusqu’à $200 \mathrm{tr} / \mathrm{mn}$.

Cet accroissement important des débits spécifiques utilisables s'explique par les meilleures caractéristiques des groupes axiaux aux fortes ouvertures. Il faut cependant également préciser, qu'à profondeur de fouilles économiquement équivalente, la contrepression sur la roue d'une turbine axiale est plus élevée que celle sur la roue de la Kaplan de même puissance nominale. Cette marge de calage permet d'utiliser la colline pour de plus forts $Q_{11}$ et donne ainsi à l'installation équipée de groupes bulbes, une meilleure productivité.

Les limites tracées représentent les caractéristiques maximales d'utilisation des groupes en fonction des variations de chute. Ces limites correspondent respectivement, en allant des hautes chutes vers les basses chutes, dans un diagramme en $\mathrm{Q}_{11}, n_{11}$;

- à la puissance maximale de l'alternateur;

- à la limite de cavitation;

- à l'ouverture maximale ou à la saturation.

Ces données, extraites des spécifications techniques des cahiers des charges, ne correspondent pas dans tous les cas aux possibilités optimales des machines, car des considérations particulières à l'installation ont pu influencer le choix des caractéristiques des machines.

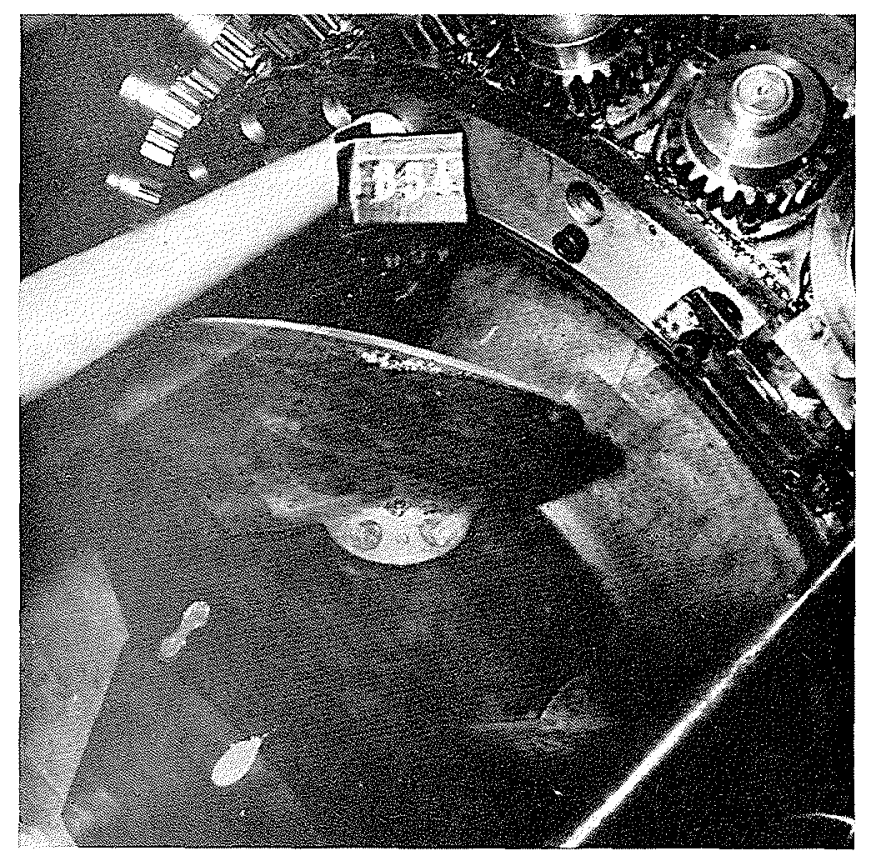

1.4/ Figure de cavitation à : haute chute - fort débit.

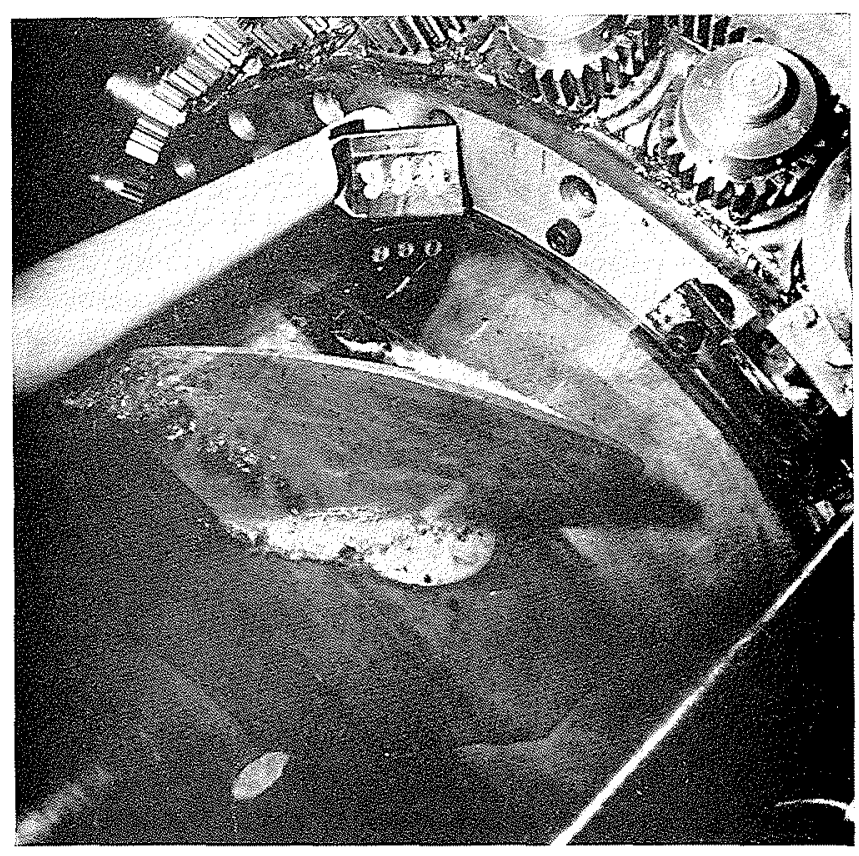

1.5/ Figure de cavitation à : basse chute - fort débit. 


\section{2. - Les différentes générations de groupes bulbes}

2.1. Groupes "bulbe-amont" de diamètre inférieur à deux mètres. Première génération.

Nous renvoyons, pour l'étude du développement de ces machines, qui constituent la première génération des groupes bulbes, au chapitre 3 du rapport de M. Cotillon. Nous analyserons ici, succinctement, les problèmes spécifiques que posèrent aux constructeurs la mise au point de ce type de groupes. Rappelons d'abord les buts recherchés:

- groupes rustiques compacts et économiques;

- ouvrages de génie civil de formes simples et économiques, liaisons génie civil matériel électro-mécanique réduites au strict nécessaire;

- fonctionnement automatique, frais d'entretien négligeables.

La conception des machines de la première génération ne posait pas de problème de limite, mais pour atteindre les buts recherchés, certaines règles de technologie, adoptées jusque-là, devaient être revues. L'inaccessibilité des organes logés à l'intérieur du bulbe et la suppression de tout appareillage de contrôle sur ces mêmes organes exigeaient, par exemple, que le fonctionnement des paliers, de la butée, des alvéoles de distribution d'huile sous pression, des joints tournants, etc., présentât une sécurité absolue. Les paliers et la butée de ces groupes comportent une pompe intégrée et un réfrigérant constitué par les parois du bulbe; ces organes répondaient exactement aux buts recherchés et ils assurent un fonctionnement sûr et autonome depuis de très nombreuses années. Les joints tournants, même pour ces

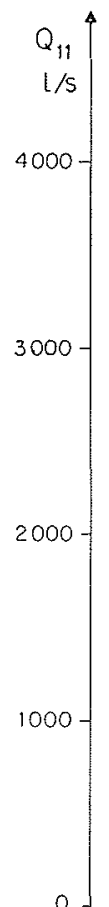

1 CADEROUSSE 2 AVIGNON 3 GERVANS 4 PIERRE BENITE 5 BEAUCAIRE 6 GAMBSHEIM S GERSTHEIM 9 LA RANCE

10 BEAUCHASTE

11 STRASBOURG

12 BOURG-LES-VALENCE

13 FRATEL

14 CAMBEYRAC

15 GOLFECH

16 RHINAU

17 BEAUMON

19 BEAUVOIR

2O SEYSSEL

20 SEYSSEL

22 VOGELGRUN

2000

$1.7 /$

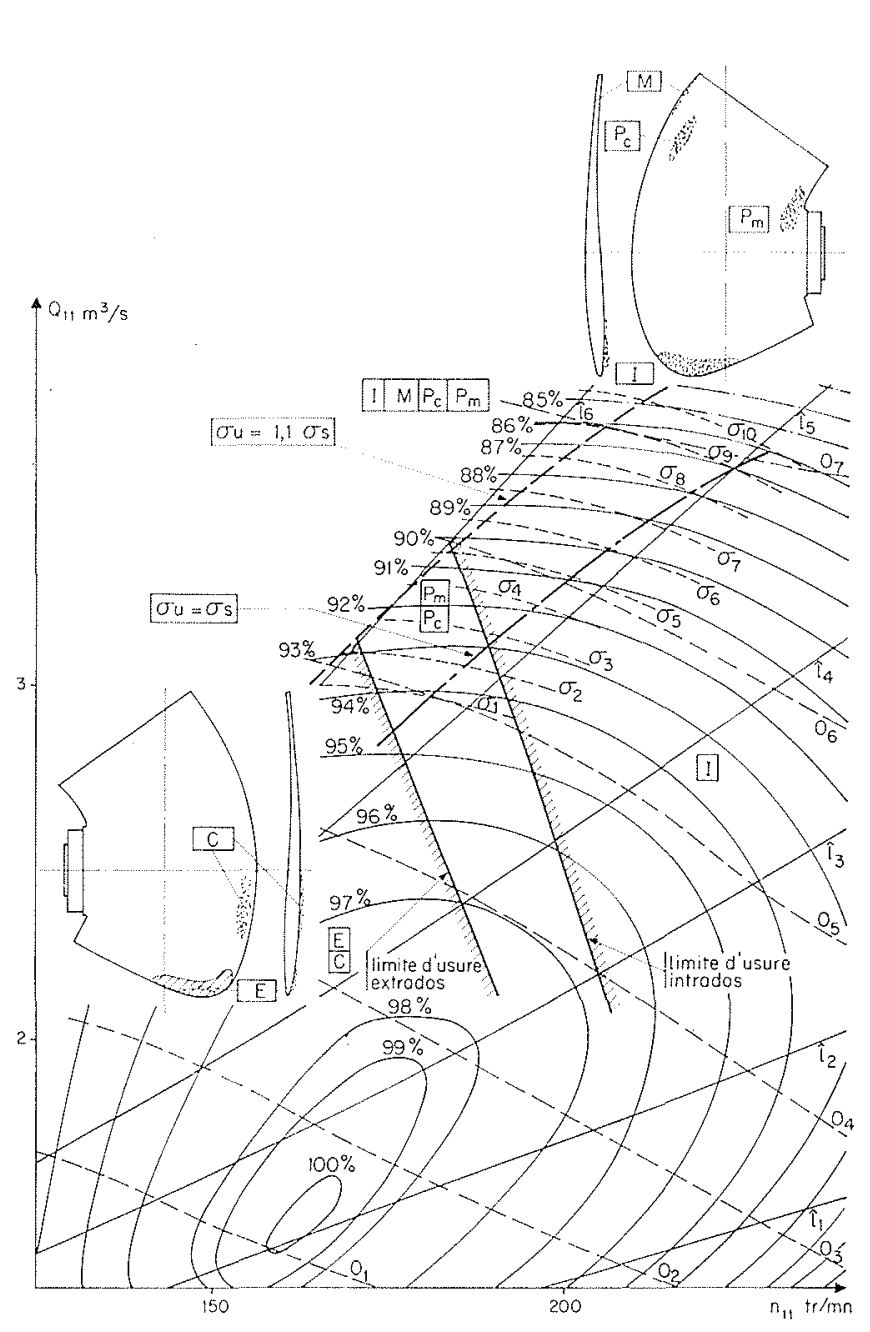

1.6/ Risques d'usure par cavitation des pales et ceinture en acier ordinaire d'un groupe bulbe de fort débit spécifique. 
machines de dimensions relativement modestes, n'offraient pas une garantie absolue. De longues recherches expérimentales et des analyses approfonldies des incidents, nous ont permis de fournir des joints tournants d'une bonne disponibilité. Les dispositions technologiques adoptées ont donc satisfait aux exigences attendues d'elles.

Précisons que le dimensionnement de ces machines conduisait, sans trop de difficultés, à des ensembles mécaniques simples, caractérisés par des fréquences propres bien plus élevées que les fréquences des forces perturbatrices d'origine hydraulique ou électromagnétique; pour tous les régimes de fonctionnement (emballements prolongés, transitoires, marche normale), les déformations des parties tournantes restaient bien inférieures à l'entrefer de l'alternateur et au jeu entre la roue et la ceinture de la turbine.

Les nombreux essais d'emballement et de déclenchements, difficiles à envisager sur des groupes classiques, ont pu être exécutés sur ces machines horizontales qui peuvent supporter un emballement continu. Ces premiers groupes bulbes constituèrent des stations d'essais exceptionnellement intéressantes pour l'analyse des vibrations, des efforts de manœuvre sur les pales, etc. Les résultats de ces mesures nous permirent d'aborder avec confiance l'étape suivante.

\subsection{Groupes «bulbe-amont" à double réglage. Deuxième génération.}

Nous considérons les bulbes amont à double réglage comme des machines de la deuxième génération; nous les diviserons en ideux groupes:

- les machines de diamètre inférieur à $4 \mathrm{~m}$;

- les machines de diamètre supérieur à $4 \mathrm{~m}$.

Cette division se justifie puisque, dans les machines du premier groupe, les problèmes d'accessibilité demeurent difficiles tandis que, dans celles du second groupe, les organes logés dans le bulbe, à l'amont ou à l'aval de l'alternateur, deviennent accessibles.

Le passage à la seconde génération s'effectua avec précaution. Les efforts hydrauliques, appliqués à la roue en porte-à-faux au cours des régimes transitoires, restaient insuffisamment connus, malgré l'ample moisson de renseignements tirés des essais sur les groupes de la première génération. Les constructeurs craignaient que, lors des transitoires et des emballements, des phénomènes particuliers se produisent tels que : cavitation à la partie haute de la roue, décollements ou poches de cavitation pulsatoires liés aux pales, susceptibles d'engendrer des vibrations et une dissymétrie importante des efforts hydrauliques.

Les conditions particulières d'exploitation de la centrale de la Rance (fermeture d'urgence des groupes, loi de débit, etc.), la rapide montée en vitesse de ces machines à faible inertie, les survitesses importantes (60 à $75 \%$ ) atteintes lors des disjonctions..., ont amené les maîtres d'œuvre à exiger que ce type de groupe puisse supporter un emballement continu. Sur les Kaplan, le peu d'essais d'emballements connus à cette époque, montraient que de fortes vibrations et des déformations importantes des parties tournantes se produisaient lors des marches à fortes survitesses; la construction de groupes bulbes, même de dimensions moyennes, ne pouvait s'envisager que si le comportement en survitesse de ces machines restait techniquement acceptable. Ces raisons expliquent la prudente croissance des dimensions des groupes axiaux et l'indécision des construc- teurs pour choisir entre les types bulbes «amont» et «aval ».

La construction des groupes de Wadrinat et de BeaumontMonteux, prototypes du genre «bulbe-amont», commença à peu près en même temps que celle des turbines-pompes axiales de Cambeyrac et d'Argentat, machines du genre «bulbe-aval ». Ces deux installations, équipées de turbines pompes, constituèrent les deux premières stations marémotrices expérimentales; le choix du type de machine, pour ces deux centrales, fut presque uniquement déterminé par l'état d'avancement de nos recherches hydrauliques et mécaniques. A cette époque, les turbines-pompes axiales, mises au point par nos services de recherches, présentaient des caractéristiques en turbine et en pompe satisfaisantes pour les deux sens d'écoulement; cependant ces performances ne pouvaient être obtenues qu'avec:

- un grande course angulaire des directrices (110 à 130');

- une roue comportant six pales réversibles (angle de manœuvre $210^{\circ}$ ).

L'adoption d'une commande des directrices par servomoteurs individuels, synchronisés par couronne et pignons coniques, permit de satisfaire à la première condition. La nécessité de prévoir des pales réversibles constituait une contrainte beaucoup plus grave, on ne pouvait plus utiliser la commande classique par bielle et manivelle, système robuste et relativement simple. Des études, longues et délicates, sur les différents types de commandes réversibles, aboutirent au choix du système pignon-crémaillère. Les énormes difficultés. rencontrées au début de ces études, nous amenèrent à rechercher des profils spéciaux de pales, pouvant être attaqués par le bord d'entrée ou de sortie, suivant le sens d'écoulement; on pensait, qu'avec ces tracés nouveaux, on pourrait réduire la course angulaire des pales à $45^{\circ}$ environ, supprimer le retournement et conserver un système de commande par bielle et manivelle.

L'incertitude de la réussite de nos recherches sur le tracé des pales non réversibles, nous contraignait donc à choisir, pour Cambeyrac, une roue comportant six pales réversibles. Le détalonnage important, exigé par le retournement des pales, interdisait l'emploi d'une roue munie de quatre pales. Le choix du tracé des pales réversibles nous conduisit à proposer au client un groupe «bulbe-aval ». De nombreuses raisons, que nous allons examiner, justifiaient cette option. Tout d'abord, nous rappelons de nouveau que nous ne connaissions pas très bien, à l'époque, les efforts hydrauliques qui pouvaient agir sur les pales de la roue pendant les régimes transitoires. Si les déclenchements, les emballements, etc., constituent pour les Kaplan verticales classiques des régimes de marche exceptionnels et si, de même, les variations de charge restent pour ces machines relativement peu fréquentes, nous savions que les conditions d'exploitation des usines marémotrices seraient beaucoup plus sévères. Les turbines-pompes marémotrices auraient à fonctionner en turbine, en pompe et en orifice pour les deux sens d'écoulements; de plus, les emballements maximaux, pour ce type de machine, atteindraient des valeurs importantes. Il fallait donc choisir un type de machine qui puisse supporter tous ces fonctionnements perturbés, sans que les parties tournantes ne viennent frotter sur les parties fixes, malgré l'entrefer réduit de l'alternateur et le faible jeu entre la ceinture et la roue. Il fallait s'assurer, de plus, que les fréquences des forces perturbatrices d'origine hydraulique resteraient toujours assez différentes des premières fréquences propres 
des parties tournantes. Ce n'était pas chose facile, d'autant plus que la réversibilité des pales conduisait à un mécanisme de commande lourd et encombrant qui augmentait, très sérieusement, le porte-à-faux de la roue, que l'on plaçât d'ailleurs ce mécanisme d'un côté ou de l'autre de la roue. La longueur et le diamètre relativement importants du moyeu (situé en définitive du côté de l'aspirateur) constituaient aussi des facteurs défavorables; en effet, une répartition non axisymétrique des pressions agissant sur le moyeu pouvait provoquer, au droit de la roue, des couples importants, amplifiés par le grand bras de levier.

Nous résumons pour conclure les raisons qui, à l'époque, nous amenèrent à proposer à E.D.F. des groupes du type «bulbe-aval» pour les stations expérimentales des usines marémotrices :

- connaissance insuffisante des forces hydrauliques pouvant se manifester lors des fonctionnements avec cavitation partielle ou généralisée;

- risques de vibrations et de déformations exagérées des parties tournantes sous l'influence de ces forces;

- nécessité de prévoir des pales réversibles, leur mécanisme cle commande entraînait une augmentation du poids de la roue et du porte-à-faux;

- conditions particulièrement sévères de fonctionnement des turbines-pompes marémotrices.

Nous verrons d'ailleurs plus loin, en étudiant les forces hydrauliques qui peuvent prendre naissance lors des régimes transitoires, que notre prudence était justifiée. Vers les années 1955-1956, nous pensions donc utiliser les groupes du type «bulbe-avall 》 pour l'équipement des usines marémotrices et ceux du type «bulbe-amont» pour les usines de rivière. Nous avions proposé ces deux types de machines à E.D.F., tout en espérant mettre au point des pales non réversibles qui pourraient permettre de généraliser le type «bulbeamont $»$.

En 1957, deux mises en route, celles de Wadrinau et Cambeyrac, constituèrent un nouveau pas en avant dans la connaissance du fonctionnement des machines axiales. Ces deux types de groupes, respectivement du genre bulbes 《amont» et «aval», comportaient des roues de 3050 et $3100 \mathrm{~mm}$ de diamètre.

Nous donnerons quelques informations sur les essais faits à Wadrinau et analyserons rapidement les résultats de ceux exécutés à Cambeyrac déjà décrits dans d'autres études [9], [10].

Les machines de Wadrinau comportent un distributeur fixe et une roue à pales mobiles; c'est la première installation, équipée de turbines axiales, qui comprenne des vannes situées à la sortie de l'aspirateur. Les mesures des efforts de manœuvre des pales, lors de leur fermeture rapide $(4 \mathrm{sec}$ environ) pendant les déclenchements, ne décelèrent aucune pulsation anormale; il faut préciser cependant que ces mesures pouvaient ne donner que des valeurs moyennes, les modulations à des fréquences de l'ordre de la vitesse de rotation risquaient d'être filtrées par les inerties et la servocommande hydraulique. Les essais de coupure, par la vanne aval, se révélèrent très satisfaisants et montrèrent que le couplage des groupes axiaux, à pales et distributeur fixes, pourrait s'effectuer très aisément. La figure 2.1 donne les principaux résultats de ces essais. Les abscisses correspondent aux levées de la vanne, donc au temps, puisque la manouvre est linéaire (temps d'ouverture de la vanne 580"). Les ordonnées, à gauche et à droite, se rapportent respec-.

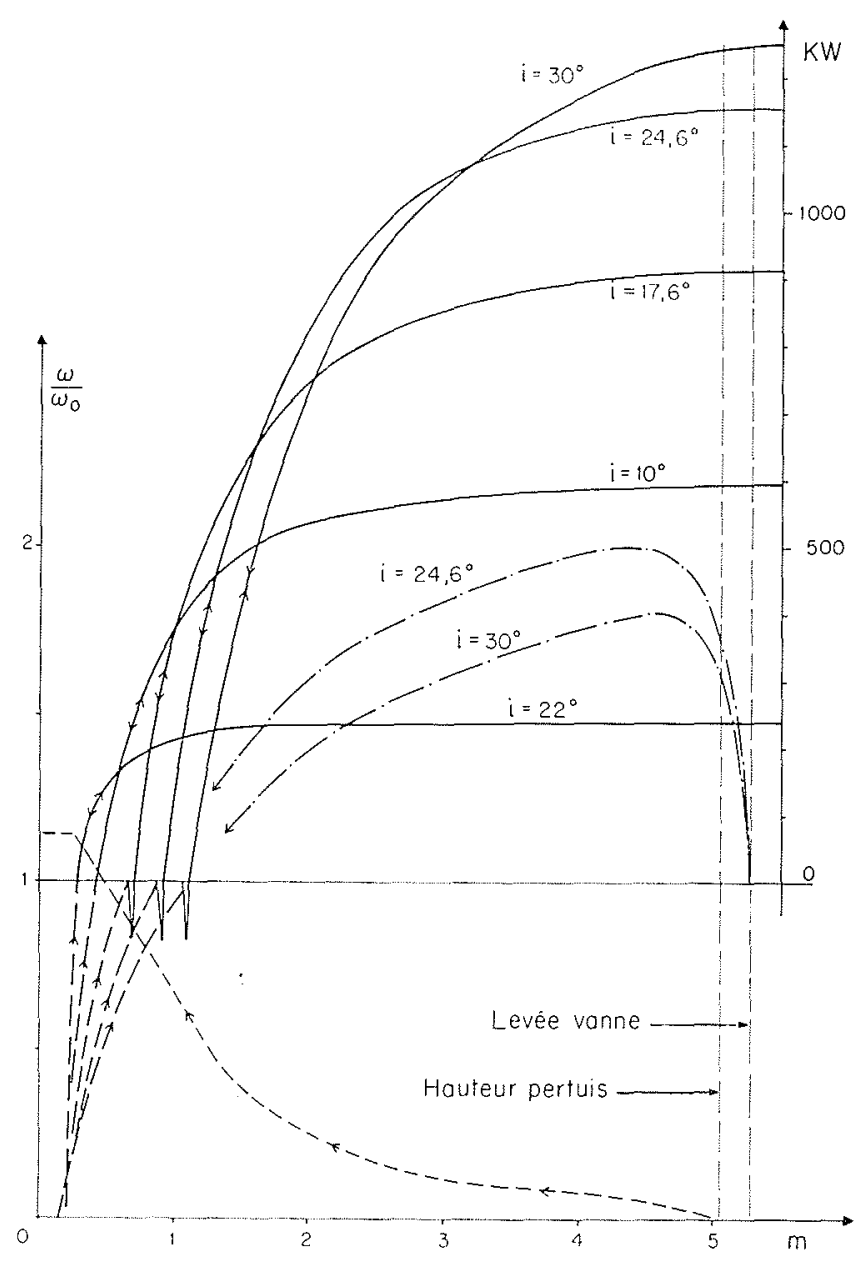

2.1/ Wadrinau.

tivement à la vitesse (par rapport au synchronisme) et à la puissance. Pour une inclinaison $i$ des pales (par exemple $30^{\circ}$ ), la vitesse, représentée par la courbe en pointillés, croit avec l'ouverture de la vanne. Au voisinage du synchronisme, le couplage asynchrone provoque une variation brusque de vitesse. Après couplage, la puissance développée par la turbine (courbe continue) augmente avec la levée de la vanne. Le couple hydraulique au démarrage croît avec l'inclinaison. Le temps nécessaire, pour atteindre le synchronisme, croît avec l'inclinaison des pales, le couplage aux fortes inclinaisons s'effectue plus aisément qu'aux faibles incidences. Précisons qu'au voisinage du synchronisme, une ouverture de la vanne de $1 \%$ provoque, respectivement, une variation de vitesse de $10 \%$ pour $i=10^{\circ}$ et $2 \%$ pour $i=30^{\circ}$. Les deux courbes en traits d'axe, relatives aux inclinaisons $30^{\circ}$ et $24,6^{\circ}$, représentent les variations de vitesse du groupe lors de deux déclenchements avec fermeture de la vanne. Ces courbes enregistrées montrent que le contrôle du débit par la vanne provoque une réduction très sensible de la vitesse d'emballement (temps 'de fermeture 'de la vanne 135').

Nos recherches sur le tracé 'des pales des turbines-pompes axiales, tracé permettant d'éviter le retournement, aboutirent, en 1956, un an avant la mise en route de Cambeyrac. 
Dès cette date, nous proposâmes à E.D.F. un groupe expérimental, du type «bulbe-amont», comme prototype des machines devant équiper l'usine marémotrice de la Rance. En 1957, le complet succès de la mise en exploitation du groupe de Cambeyrac, facilité par l'utilisation d'un important appareillage de mesures, nous apporta une série de renseignements de premier ordre concernant le fonctionnement des turbo-machines axiales lors des régimes transitoires correspondant aux différentes marches en turbine et en pompe, pour les deux sens d'écoulement. Nous renvoyons le lecteur aux publications existantes [9], [10], [11], [12], [13], pour l'analyse de ces résultats, sans la connaissance desquels les études du groupe de Saint-Malo auraient présenté de très grandes difficultés. Nous ne reprendrons pas, non plus, l'examen des problèmes posés par la conception et la fabrication du groupe de Cambeyrac. Nous reproduisons le des$\sin$ du moyeu sur la figure 2.2. Cette coupe montre que la roue devait nécessairement être supportée par deux paliers situés, respectivement, à l'aval et à l'amont des pales.

Nos études sur les groupes «bulbe-amont» de grandes dimensions commencèrent en 1957, tandis que se poursuivait la fabrication des groupes de Beaumont-Monteux et d'Argentat. En 1958, la mise en exploitation de la machine
d'Argentat, du type «bulbe-aval», s'effectua dans les meilleures conditions. Ce groupe développe $14,4 \mathrm{MW}$; il fonctionne, en turbine, sous une chute de $16,5 \mathrm{~m}$ et refoule, en pompe, dans le réservoir amont, sous une hauteur de $11 \mathrm{~m}$. Ce groupe reste encore aujourd'hui la turbine-pompe axiale la plus puissante; il comporte une roue de $3800 \mathrm{~mm}$ de diamètre.

Toujours en 1958, avec la mise en route du groupe de Beaumont-Monteux, commençait la série des «bulbes. amont $\gg$ de dimensions respectables, puisque le diamètre de roue atteint $3800 \mathrm{~mm}$. Cette machine fonctionne de façon continue depuis son démarrage; elle a dépassé très largement les $100000 \mathrm{~h}$ d'exploitation et donne entière satisfaction. Les dimensions moyennes de ces machines ne permettent pas de prévoir une bonne accessibilité pour certains organes logés à l'intérieur du bulbe, c'est le cas du palier turbine et du joint tournant. Les résultats d'exploitation obtenus montrent que la conception technologique, et la fabrication soignée de ces parties de la machine, ont permis d'atteindre les buts visés. Nous n'analyserons pas les résultats des mesures faites sur le groupe de Beaumont-Monteux lors de sa mise en exploitation. L'essai à l'emballement maximal pendant près d'une heure nous paraît digne d'être signalé. Après cette

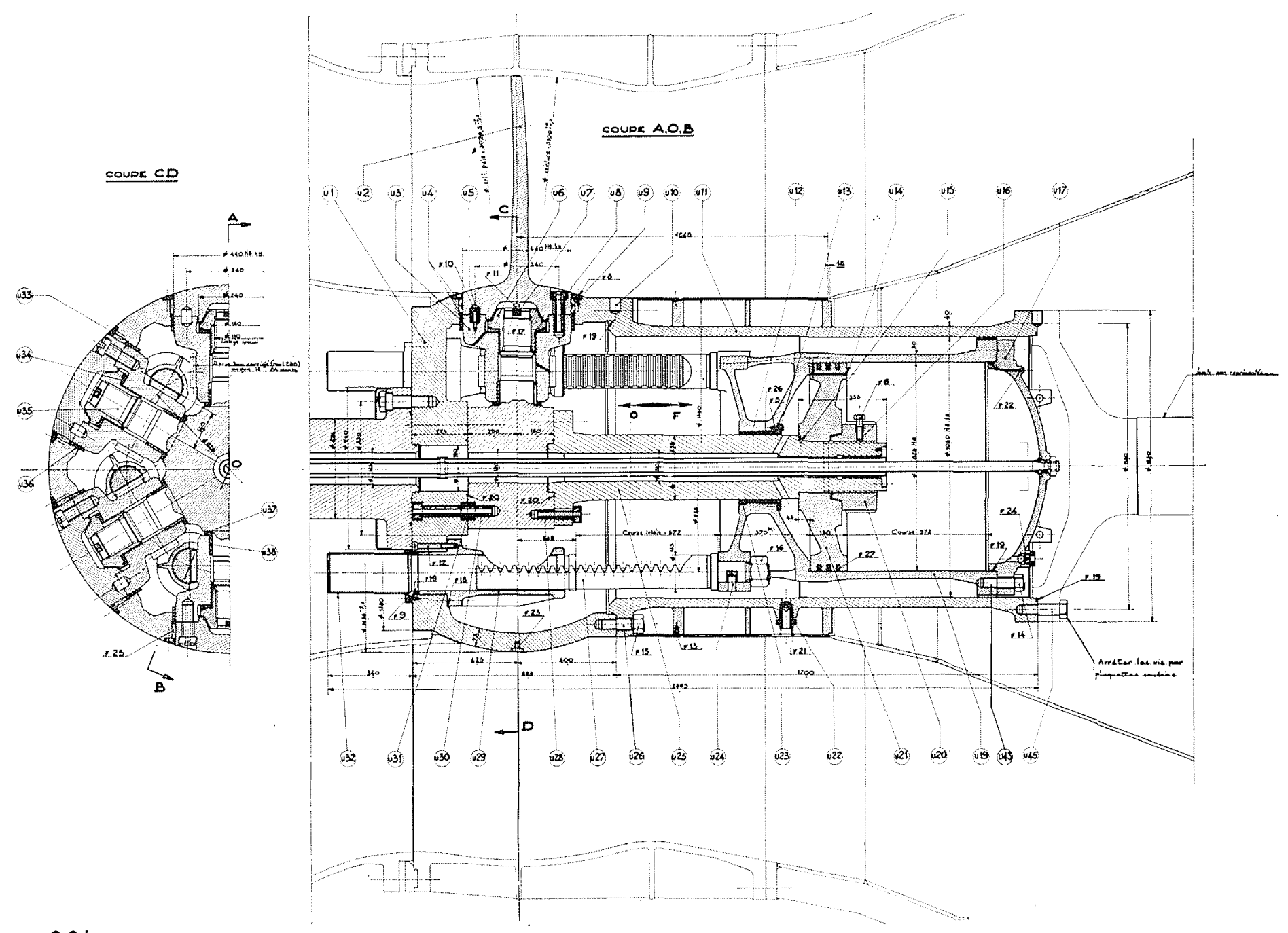

2.21 
épreuve extrêmement sévère, supportée par la machine sans aucun dommage, nous pouvions envisager avec confiance la mise au point du groupe de Saint-Malo, à l'époque en cours de fabrication et de montage.

En 1959, la mise en route du groupe marémoteur expérimental de Saint-Malo, du type «bulbe-amont» avec roue en porte-à-faux, vint couronner plus de dix ans d'efforts. Nous renvoyons le lecteur aux différentes publications parues [13], [14] qui traitent des problèmes posés par la conception et la fabrication. Le succès de l'exploitation de cette machine, munie d'une roue de $5,800 \mathrm{~m}$ de diamètre, prouva que l'utilisation des groupes axiaux de ce type pouvait s'envisager pour l'équipement des usines marémotrices et des grands fleuves français.

En 1966, Pierre-Bénite ouvrait la carrière des groupes bulbes de rivière, la Rance celle des turbines-pompes axiales pour les usines marémotrices. De nombreux articles se rapportent à ces deux installations [2], [15], [16], articles auxquels nous renvoyons le lecteur.

\subsection{Groupes bulbes simplifiés. Troisième génération.}

Avant d'aborder la dernière génération, celle des groupes bulbes à pales et distributeur fixes, nous croyons utile de résumer quelques-uns des essais les plus marquants effectués sur des machines axiales de grandes dimensions. Nous avons souvent insisté sur l'importance de la bonne tenue mécanique des groupes bulbes lors des régimes transitoires; c'est une condition impérative. L'idée très ancienne d'utiliser des groupes axiaux, du type hélice, n'a pas déclenché, dès sa naissance, un développement rapide de ce type de machines. Ce décalage entre l'idée et les réalisations s'explique par le fait que les constructeurs craignaient que ces turbines ne soient le siège de vibrations dangereuses lors des régimes transitoires. Alsthom-Neyrpic a frayé la voie, les constructeurs qui l'ont suivie ont été confrontés aux mêmes difficultés dès qu'ils ont voulu fabriquer des groupes de grandes dimensions. C'est ainsi que L.M.Z., le constructeur russe de Leningrad, a jugé bon de réaliser deux prototypes de conception différente pour l'usine de Saratov. L'une de ces machines possède quatre paliers, situés respectivement de part et d'autre du rotor de l'alternateur et de la rone de la turbine; l'un des paliers de la turbine se trouve donc logé dans un croisillon placé à l'entrée de l'aspirateur, cette disposition supprime le porte-à-faux. L'autre groupe expérimental comporte trois paliers, la roue de $7,5 \mathrm{~m}$ de diamètre est en porte-à-faux. Ces deux machines développent chacune $47,3 \mathrm{MW}$ sous $10,6 \mathrm{~m}$ de chute et tournent à $75 \mathrm{tr} / \mathrm{mn}$ [17]. Le fait que ce constructeur ait réalisé ces deux prototypes montre qu'il était préoccupé par les problèmes posés par le porte-à-faux.

Précurseurs, exposés donc aux échecs les plus graves, nous croyons utile d'examiner succinctement comment nous avons résolu les problèmes soulevés par la conception et la fabrication des groupes bulbes de la deuxième génération, c'està-dire de machines axiales de grandes dimensions à double réglage.

Les premières mesures précises, sur la tenue mécanique des parties tournantes et des supports, furent exécutées sur la machine expérimentale de Saint-Malo [14]. Rappelons que nous poursuivions trois buts principaux:

- préciser les conditions de fonctionnement des paliers supportant des charges supérieures à $100 \mathrm{t}$ et tournant à des vitesses variant de 75 à $100 \mathrm{tr} / \mathrm{mn}$ en marche nor- male (plus de trois fois plus vite à l'emballement), nous cherchions la confirmation des métho'des de calcul pour : l'épaisseur du film d'huile, les répartitions des températures, les déformations, le système de réfrigération, etc.;

- rechercher les fréquences propres les plus basses des supports, mesurer leurs déformations maximales, comparer ces valeurs aux résultats des calculs;

- analyser les déplacements des parties tournantes, en marche normale et lors des transitoires.

Les deux premières séries de recherches aboutirent rapidement et montrèrent que le calcul permettait de dimensionner, de façon suffisamment précise, les organes porteurs et les supports du groupe. L'analyse des mouvements des parties tournantes se révéla beaucoup plus délicate. Les déplacements verticaux lors des transitoires restaient, bien entendu, limités par le jeu du palier turbine; la roue se soulevait sous l'influence des efforts hydrauliques et venait heurter la partie supérieure du coussinet, puis reposait de nouveau sur le film d'huile. Dans les groupes verticaux, les efforts radiaux nécessaires pour accélérer les parties tournantes restent négligeables par rapport aux forces perturbatrices (d'origine hydraulique ou électrique). Pour une machine à axe vertical, des déplacements de l'arbre dans les coussinets de l'ordre du jeu n'impliquent donc pas, en général, l'existence d'efforts importants. Le soulèvement des parties tournantes d'un groupe bulbe pendant les régimes transitoires prouve que les forces hydrauliques, appliquées au droit de la roue, peuvent atteindre et même dépasser $100 \mathrm{t}$ pour des roues de l'ordre de $6 \mathrm{~m}$ de diamètre.

Quelques expérimentateurs ont constaté, lors de fonctionnements avec cavitation partielle, des déformations inexpliquées de l'arbre au voisinage de la roue de certaines turbomachines à axe horizontal [18]. D'après M. Rosenmann, les forces perturbatrices seraient provoquées par un déséquilibre hydrodynamique induit par la cavitation. Des jauges placées sur l'arbre lui permettraient de mesurer les couples et les efforts. Les forces observées variaient avec les régimes de marche, elles pouvaient être de direction fixe par rapport à la roue ou sauter "de pale en pale.

L'importance des investissements envisagés pour l'équipement de l'usine de la Rance et des centrales du Rhône et du Rhin, justifiait la mise au point d'un appareillage délicat, capable de mesurer les efforts hydrauliques pulsatoires pouvant agir sur la roue lors des régimes transitoires. L'équipement de mesures d'E.D.F. permit de faire des mesures sur la roue d'un des groupes de la Rance [19]; notre appareillage vient de servir aux essais sur les pales d'une des machines de Gervans.

Avant d'analyser les essais effectués sur les roues des turbines, nous donnerons, pour les régimes transitoires, quelques résultats de mesures caractéristiques concernant:

- les variations des pressions le long des parois intérieure et extérieure des conduits hydrauliques;

- l'évolution de la vitesse de la machine;

- la variation des poussées axiales et radiales sur les supports;

- les déplacements des parties tournantes.

De nombreux essais de ce genre permirent, à la mise en route des groupes de Pierre-Bénite, d'étudier les phénomènes vibratoires lors des régimes transitoires. Nous examinerons ici les expériences, faites en 1970, sur les machines de Beaucaire qui comportent des roues de $6,250 \mathrm{~m}$ de 
diamètre. Les enregistrements effectués pendant les premiers déclenchements à fortes puissances, dévoilèrent l'existence de pulsations de pression assez élevées accompagnées de bruits sourds. Ces coupures d'urgence correspondaient à des fermetures linéaires du distributeur (25" environ) et des pales. Les déclenchements, avec passage en déchargeur, se caractérisaient par des phénomènes vibratoires moins sévères; la fermeture simultanée de la vanne et des organes de réglage constituait donc un moyen de réduire ces surpressions. Client et constructeur décidèrent toutefois d'analyser, de manière plus approfondie, les phénomènes vibratoires qui se manifestaient lors des déclenchements. L'intervention de la vanne lors des déclenchements, n'était pas toujours envisageable, ce qui nous obligeait donc à trouver des moyens pour réduire l'amplitude de ces pulsations de pression parasites, par exemple en recherchant des lois de manœuvre mieux adaptées ou en injectant de l'air comprimé au droit du cône turbine.

La figure 2.3 correspond à un déclenchement à partir de $22,2 \mathrm{MW}$ avec des lois de fermetures linéaires et sans intervention de la vanne aval. En marche normale, avant le déclenchement, les pressions mesurées par les capteurs $\mathbf{P}_{1}$, $\mathrm{P}_{2}, \mathrm{P}_{3}$ et $\mathrm{P}_{4}$ (voir fig. 2.4) oscillent principalement ì là fréquence de passage des pales, soit $6,25 \mathrm{~Hz}(1,5625 \times 4)$. Les amplitudes détectées par les capteurs $P_{1}, P_{2}$ et $P_{3}$ restent négligeables, tandis que celles mesurées en $P_{4}$, à l'aval de la roue et au point haut de l'aspirateur varient de $\pm 2 \mathrm{~m}$ et révèlent donc la présence de sillages liés aux pales. Après le déclenchement, en $P_{3}$ et $P_{4}$, les amplitudes de pulsations de pressions croissent lors de la montée en vitesse jusqu'à la survitesse maximale où elles atteignent respectivement $\pm 7 \mathrm{~m}$ en $\mathrm{P}_{3}$ et $\pm 4 \mathrm{~m}$ en $\mathrm{P}_{4}$; à l'amon't de la roue, en $P_{1}$ et $P_{2}$, les variations restent faibles et comparables à celles constatées en marche normale. En régime d'accélération, la roue fonctionne toujours en turbine, lorsque la vitesse croît les sillages liés aux aubes s'épaississent et induisent des écoulements très turbulents à l'aval de la roue, mais il n'apparaît pas d'instabilité globale. La survitesse maximale correspond à un régime d'emballement, la roue ne fournit alors aucune énergie au fluide. L'accélération décroît de façon continue lors de la montée en vitesse; il ne se produit pas de variation brutale de couple, ce qui caractérise une suite id'écoulements relativement stables.

Toujours lors de la montée en vitesse, vers l'entrée des pales en $\mathrm{P}_{5}$ et à leur sortie en $\mathrm{P}_{6}$, les pressions pulsatoires mesurées décèlent la présence des sillages liés aux aubes (voir fig. 2.5). En $P_{5}$, les pressions oscillent fortement, les minima atteignent le vide pour une gamme de vitesse centrée autour de la survitesse maximale; lorsque deux pales consécutives se trouvent de part et d'autre du point haut, les sillages n'obstruent donc pas complètement le canal qu'elles limitent. En $\mathrm{P}_{6}$, à la sortie de la roue, la pression se maintient au vide, ce qui prouve que les sillages bouchent en moyenne complètement le canal. Une poche de vide se forme donc, à la partie haute du conduit, vers la sortie des aubes, pour une gamme de vitesse centrée autour de la vitesse maximale; cette poche persiste pendant 5 à $6 \mathrm{~s}$.

Au-delà de la vitesse maximale, en régime de freinage, apparaissent des instabilités d'écoulement qui se manifestent par des pulsations de pressions sensibles à l'amont, en $P_{1}$, $\mathrm{P}_{2}$, et importantes à l'aval, en $\mathrm{P}_{3}, \mathrm{P}_{4}$. Ces instabilités globales provoquent des changements brusques de couples, donc des échanges de quantité de mouvement, phénomènes que révèlent les variations d'accélérations constatées sur la courbe des vitesses.
Examinons ce qui se passe dans la zone de freinage. Au-delà de la vitesse maximale, l'ouverture $O$ du distributeur continue à décroître, tandis que l'inclinaison des pales $i$ reste pratiquement constante. A des $\mathrm{O}_{1}$ et $i_{1}$ donnés, et sous la chute d'essais, correspond une vitesse d'emballement bien définie $n_{\mathrm{e}}\left(\mathrm{O}_{1} i_{1}\right)=n_{\mathrm{e} 1}$. Lorsque l'ouverture du distributeur atteint la valeur $\mathrm{O}_{1}$, la vitesse $n_{1}$ du groupe, à cause de l'inertie du groupe, reste supérieure à la vitesse d'emballement $n_{c 1}$. La roue fonctionne donc en pompe accélératrice en absorbant progressivement l'énergie cinétique du groupe; plus la différence $n_{1}-n_{e 1}$ est grande, plus la machine tendra à accélérer le débit. Ces marches en pompe accélératrice ont été étudiées, à notre demande, par le laboratoire de Chatou. On constate deux principaux types d'écoulements:

- fonctionnement du type emballement, avec décollements sur l'intrados et l'extrados des pales et une torche au-delà de l'ogive;

- fonctionnement du genre pompe axiale à faible débit, caractérisé par un rouleau à l'entrée vers la paroi extérieure, un rouleau à la sortie entourant l'ogive et un écoulement centrifuge dans la roue.

Le passage d'un type d'écoulement à l'autre peut provoquer ide fortes variations 'de débit.

Il est difficile de définir à partir des essais, lors d'un déclenchement, la hauteur de refoulement développée par la machine. Les pressions à l'amont et à l'aval de la roue pulsen't très fortement, parfois leur différence doit dépasser la hauteur à débit nul de la roue et provoquer de fortes variations de débit.

Ces régimes perturbés se poursuivent tant que la vitesse du groupe $n(\mathrm{O}, i)$ reste supérieure à la vitesse d'emballement $n_{c}(\mathrm{O}, i)$. On peut réduire cet écart $n(\mathrm{O}, i)-n_{e}(\mathrm{O}, i)$ dès le début de la zone de freinage:

- soit en réduisant la vitesse de fermeture du distributeur au-delà de la vitesse maximale;

- soit en augmentant celle 'des pales;

- soit par une combinaison des deux.

Lors des déclenchements, on constate différents types de pulsations de pressions :

- Des oscillations de fréquence voisines de celle du passage des pales, soit $6,25 \mathrm{~Hz}$. Elles se produisent plus souvent lors des montées en vitesse, mais aussi en période de freinage. Les amplitudes maximales correspondent au point haut du conduit.

- Des oscillations en phase sur tous les capteurs d'amplitude et de fréquence, variant respectivement de 10 à $20 \mathrm{~m}$ et de 2 à $5 \mathrm{~Hz}$. Elles n'existent que dans la zone de freinage où se produisent 'des instabilités globales d'écoulement.

- Des oscillations déphasées sur les différents capteurs, révélant des écoulements du genre torche.

- Des pulsations brusques et d'amplitudes importantes, en phase sur tous les capteurs. Elles correspondent, soit à des résorptions brutales de la poche de vide apparaissant à la partie haute de l'aspirateur, soit à des résonances.

Rappelons que la fonction de transfert, qui relie les variations de pression et de débit pour une conduite à caractéristique unique, se définit par:

$$
\mathrm{H}(\omega) / \mathrm{V}(\omega)=i \frac{a \mathrm{~V}_{0}}{g \mathrm{H}_{o}} \operatorname{tg}(\omega \mathrm{L} / a)
$$

oit $\omega, \mathrm{L}, a$, représentent respectivement la pulsation de la 


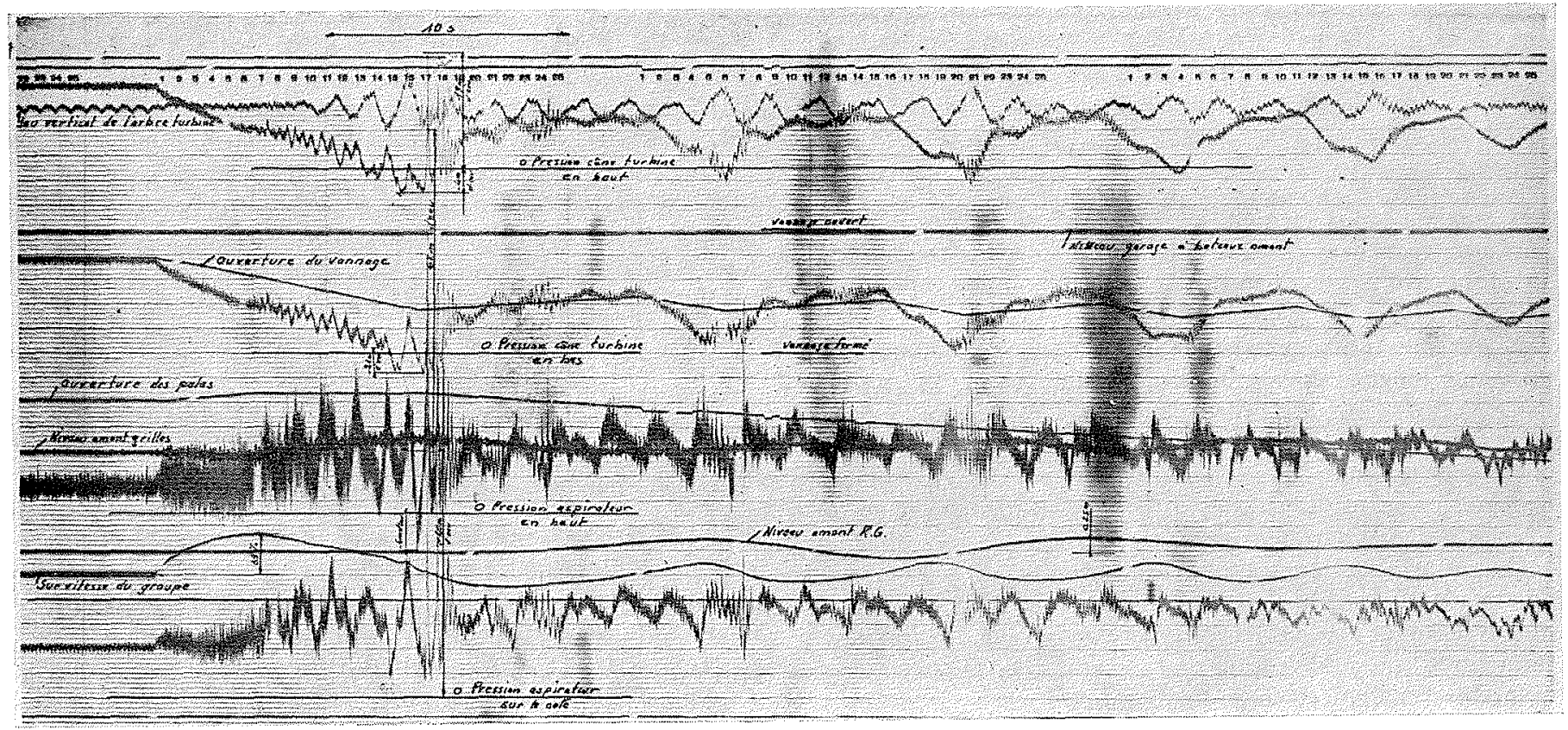

$2.3 /$

\section{$2.5 /$}
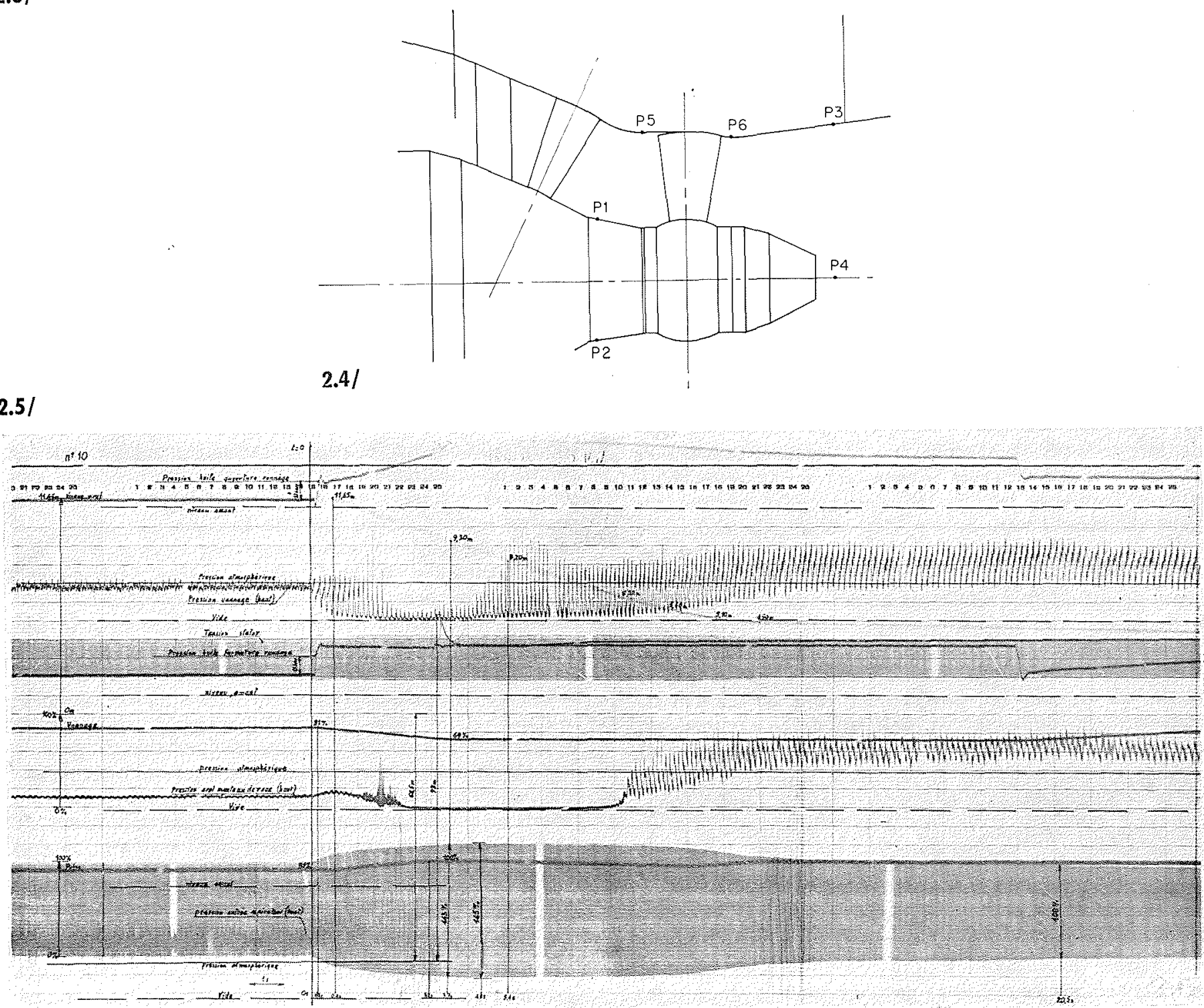
perturbation forcée, la longueur de la conduite et la célérité des ondes. La résonance se produit donc pour les pulsations $([2 n+1] / 2) \pi=(\omega \mathrm{L}) / a$, soit pour les fréquences:

$$
f=(2 n+1) \frac{a}{4 \mathrm{~L}}=\frac{2 n+1}{\theta} \operatorname{avec} \theta=4 \mathrm{~L} / a \text {. }
$$

Les maxima, pour les débits, peuvent correspondre d'autre part aux fréquences $f=2 n / \theta$. Les deux suites de fréquences se rapportent respectivement aux harmoniques impairs et pairs de $1 / \theta$.

La période fondamentale $a / 4 \mathrm{~L}$ de l'ensemble du conduit, pour une longueur totale de $65 \mathrm{~m}$ et une célérité $a$, voisine de $1000 \mathrm{~m} / \mathrm{s}$, est de l'ordre de $4 \mathrm{~Hz}$. L'étude des phénomènes de coup de bélier d'onde dans des conduits de formes complexes, et pour des écoulements comportant des poches de vide, présente de grandes difficultés; la définition de la célérité 'des ondes reste assez approximative.

Ces essais ont montré que les phénomènes pulsatoires, qui se manifestent lors des déclenchements, sont beaucoup moins sensibles lors des passages en déchargeur que lors des fermetures sans intervention de la vanne aval. Deux actions favorables expliquent cette amélioration:

- une limitation de fermeture du vannage intervient au voisinage de la vitesse maximale; elle diminue l'écart $n_{1}-n_{c 1}$,

- une remontée des pressions à l'aval de la roue, qui réduit la poche de vide qui se produit au point haut de l'aspirateur à la sortie des aubes.

Les déclenchements normaux s'effectuent toujours par passage en déchargeur pour bénéficier des effets favorables de ce type de fermeture.

Pour améliorer les déclenchements exceptionnels, qui n'interviennent que lors d'un incident sur la vanne, nous avons étudié l'influence:

- des lois de manœuvre du vannage et des pales;

- d'une injection d'air.

Bien que l'amélioration apportée par une injection d'air soit importante, nous avons adopté une solution d'efficacité équivalente mais de réalisation pratique plus simple. Nous prévoyons une fermeture du distributeur relativement rapide, lors de la période d'accélération du groupe, pour limiter la survitesse. Au début de la zone de freinage, nous réduisons suffisamment la vitesse de fermeture du distributeur pour diminuer l'écart $n_{1}-n_{c 1}$ et, partant, le couple de freinage. La réalisation de la loi de mancuvre n'offre pas de difficulté, car la durée de la période d'accélération reste à peu près constante pour toutes les conditions de fermeture à envisager.

Maître d'œuvre et constructeur prévirent, de longue date, la campagne de mesures à effectuer sur l'un des groupes de l'usine de Gervans qui possède une roue de $6,250 \mathrm{~m}$ de diamètre. Lors de la mise en exploitation de cette machine, nous poursuivions deux buts principaux :

- mesurer les contraintes pulsatoires agissant sur les pales, en régimes permanent et transitoire, et contrôler qu'elles ne compromettraient pas la tenue à la fatigue des groupes bulbes sans réglage PF-DF;

- 'définir les conditions optimales d'exploitation des groupes PF-DF.

Une étude très importante avait déjà été réalisée sur l'un des groupes de l'usine de la Rance [19], nous y renvoyons le lecteur. Nous ne décrirons pas l'appareillage de mesure, qui ne diffère pas sensiblement de celui utilisé sur le groupe AN 3 de la Rance; nous nous limiterons à l'analyse des résultats significatifs concernant les contraintes pulsatoires en régime permanent, en marche déconjuguée et en régime transitoire.

Les fluctuations des contraintes, mesurées lors des fonctionnements à faible puissance (inférieure à $10 \mathrm{MW}$ ), se caractérisent par une fréquence fondamentale égale à celle de la rotation $(6,25 / 4 \mathrm{~Hz})$. Les amplitudes restent faibles, de l'ordre de $1 \mathrm{hb}$. A partir de $10 \mathrm{MW}$, des pulsations à fréquences plus élevées apparaissen't, notamment dans la gamme des 130 à $150 \mathrm{~Hz}$, gamme dans laquelle se situe la fréquence qui correspond au quatrième mode de vibration de la pale.

Les quatre premières fréquences propres de la pale, mesurées 'dans l'air atteignent respectivement:

$1^{\mathrm{re}}, 40 \mathrm{~Hz}$, mode de flexion avec encastrement au droit du plateau;

$2^{\circ}, \quad 60 \mathrm{~Hz}$, mode de torsion autour d'une ligne modale à peu près radiale;

$3^{\circ}, \quad 95 \mathrm{~Hz}$, mode couplé complexe;

$4^{\mathrm{a}}, 140 \mathrm{~Hz}$ environ, mode couplé complexe.

Précisons que toutes les mesures de pression, à l'amont ou à l'aval de la roue, montrent l'importance de la fréquence $6,25 \mathrm{~Hz}$ liée au passage des quatre pales. Les aubes voient, d'autre part, défiler les 24 directrices, on peut donc penser que l'amplitude des pulsations de pression, de fréquence $24 \times 6,25=150 \mathrm{~Hz}$ n'est pas complètement négligeable. Cependant, cette fréquence de $150 \mathrm{~Hz}$ reste assez éloignée de la fréquence propre correspondant au quatrième mode de vibration de la pale vibrant dans l'eau; ces perturbations, à $150 \mathrm{~Hz}$, ne peuvent donc pas exciter des pulsations de contrainte dans la pale.

Remarquons que le temps que met une particule fluide pour parcourir le profil de la pale, reste de lordre de $0,1 \mathrm{~s}$. $\mathrm{La}$ roue doit donc constituer un filtre puissant pour des perturbations de fréquence bien supérieures à $10 \mathrm{~Hz}$. C'est le cas des harmoniques de la vitesse de rotation se situant dans la bande des 130 à $140 \mathrm{~Hz}$. Les pulsations de contraintes de fréquences voisines 'de 130 à $140 \mathrm{~Hz}$, que l'on mesure sur la pale, sont donc très probablement excitées par des forces perturbatrices prenant naissance au voisinage des profils eux-mêmes. Ces excitations, d'origine hydraulique, peuvent se produire au droit de décollements apparaissant en un point quelconque du profil.

Les fluctuations maximales des contraintes se produisent, en général, au droit de l'encastrement de la pale sur le plateau. A la puissance de $20 \mathrm{MW}$, les oscillations à la fréquence de rotation restent prépondérantes, mais celles à $140 \mathrm{~Hz}$ deviennent relativement importantes. Autour d'une contrainte moyenne de $5 \mathrm{hb}$, les pulsations totales atteignent $\pm 2,5 \mathrm{hb}$. A $27,4 \mathrm{MW}$, les amplitudes des deux types de vibrations sont comparables, autour d'une valeur moyenne de $6 \mathrm{hb}$ on mesure ides fluctuations totales de $\pm 3 \mathrm{hb}$ environ. Pour 32,1 MW les pulsations à la fréquence de rotation redeviennent prépondérantes, autour d'une contrainte de $6 \mathrm{hb}$, les oscillations ne sont alors que de $\pm 1 \mathrm{hb}$. Enfin, pour $34 \mathrm{MW}$ les fluctuations à la fréquence de rotation restent prépondérantes, la contrainte moyenne atteint presque $7 \mathrm{hb}$, mais les pulsations diminuent encore jusqu'à $\pm 0,8 \mathrm{hb}$.

L'analyse du comportement de la machine, lors de régimes fortement déconjugués, ne révèle pas d'accroissement sen- 
sible des amplitudes des contraintes moyennes et pulsatoires par rapport à celles mesurées en marche normale. Les amplitudes maximales se manifestent pour une gamme de puissances bien limitée et bien définie, la même pour les fonctionnements normaux et pour les régimes déconjugués.

Les contraintes mesurées lors des 'déclenchements restent très acceptables. Les perturbations les plus visibles prennent naissance pendant la période de freinage. Les pulsations de contraintes sur les aubes et les variations du couple de freinage se manifestent simultanément, mais ne possèdent pas en général la même fréquence. Les efforts hydrauliques, qui tendent à soulever les parties tournantes de la machine, apparaissent surtout lors de la décélération du groupe. Ces efforts sont détectés par la mesure des déplacements verticaux de l'arbre par rapport au coussinet du palier situé immédiatement à l'amont de la roue.

Cette nouvelle et importante campagne d'essais, effectuée sur un des groupes de Gervans, montre que la tenue des machines bulbes, lors des transitoires, ne soulève pas de grande difficulté si les lois de manœuvre des organes de vannage sont judicieusement choisies. Ces mesures nous ont aussi permis de connaître, de façon plus précise, les efforts hydrauliques qui agissent sur les pales penidant les régimes permanent, conjugué, déconjugué et transitoire. Ces données de base plus exactes faciliteront la détermination des coefficients de sécurité à adopter pour le dimensionnement des organes principaux des groupes bulbes de grandes puissances. Enfin, ces expériences prouvent que la mise en exploitation des groupes bulbes, à pales et distributeur fixes, peut être envisagée avec grande confiance. En vue de cette mise en route prochaine, des essais complets sur les transitoires des groupes bulbes PF-DF ont aussi été exécutés.

Nous avons déjà décrit succinctement les essais de coupure, par la vanne aval; 'du groupe de Wadrinau dont la roue fait seulement $3050 \mathrm{~mm}$ de diamètre. Les roues des machines PF-DF de Sauveterre atteignent $6900 \mathrm{~mm}$ de diamètre, nous avons donc jugé utile de refaire des essais sur le groupe de Gervans de $6250 \mathrm{~mm}$ de diamètre.

Nous n'étudierons pas les groupes du type Sauveterre, analysés par M. Beslin, ingénieur à la C.N.R., dans son rapport: "Idées actuelles sur la conception des groupes bulbes. Exemple de Caderousse », nous appuierons seulement sur quelques-unes des particularités techniques de ces machines. La fixation des aubes, sur le moyeu d'une roue hélice à pales fixes, soulève des difficultés moindres que celles posées par la conception mécanique d'une roue hélice à pales mobiles. L'absence de jeu, le dimensionnement relativement large des accouplements, etc., confèrent aux roues hélices à pales fixes, une plus grande aptitude à supporter des contraintes pulsatoires élevées. La grande rigidité de la ligne d'arbre, la réduction du poids de la roue et donc de la charge sur le palier turbine constituent des éléments favorables. Enfin, le distributeur à aubes fixes forme, dans son ensemble, un support de très grande raideur. Pour ce type de groupe, les déformations des pièces mobiles et fixes resteront faibles sous l'effet des forces d'origine hydraulique et électromagnétique, tandis que les fréquences propres seront assez hautes.

Les essais de transitoires sur le groupe de Gervans, avec pales et distributeur bloqués à des ouvertures correspondant aux caractéristiques des machines de Sauveterre, montrent que les phénomènes pulsatoires ne sont pas plus défavorables que ceux mesurés lors des coupures d'un groupe à double réglage. Au contraire, la fermeture d'un groupe PF-DF par la vanne aval semble moins perturbée : les pressions évoluent plus régulièrement, les torches n'apparaissent pratiquement pas lors idu freinage, les déplacements radiaux au droit du palier turbine sont plus réduits.

En ce qui concerne le 'démarrage et la prise de charge, signalons que la montée en vitesse se fait progressivement et que le couplage s'effectue dans ide bonnes conditions.

Nous dirons enfin quelques mots sur la coupure d'urgence par la vanne amont. Ce type de fermeture provoque des écoulements beaucoup plus perturbés que ceux qui se produisent lors d'une coupure par la vanne aval. Il faut n'y recourir qu'après avoir épuisé toutes les autres possibilités. Il serait inutilement risqué de fermer la vanne amont à la suite d'incidents réparables assez rapidement, incidents se rapportant, par exemple, aux composants du système de commande te la vanne aval. Les essais sur modèle révèlent des déséquilibres importants dans les écoulements à l'emballement, au droit de la roue; ces déséquilibres s'accentuent lors 'd'une coupure amont. Les phénomènes, liés au fonctionnement de la roue en pompe accélératrice lors du freinage, seront moins défavorables vu la longueur du temps de fermeture de la vanne amont.

\section{3. - Conclusions et perspectives}

Depuis Castet, mis en route en 1953, jusqu'à Caderousse, en cours de fabrication, en passant par les centrales équipant le Rhône, le Rhin, la Garonne et l'usine marémotrice de la Rance, la réussite des groupes bulbes s'avère éclatante. La plus grande partie des 212 groupes bulbes ( 74 de plus de $5 \mathrm{~m}$ de diamètre) fabriqués par les constructeurs français du groupe N-CL fonctionnent de façon satisfaisante, tandis que quelques-unes de ces machines se trouvent en cours de fabrication ou de montage; la division Neyrpic d'Alsthom en a contruit 125 environ, dont 57 de plus de $5 \mathrm{~m}$ ide diamètre de roue. La puissance unitaire maximale en fonctionnement revient aux machines de Beaucaire, avec $35 \mathrm{MW}$ pour un diamètre de roue de $6250 \mathrm{~mm}$ et une chute maximale de $15,3 \mathrm{~m}$. Les deux groupes de Sauveterre, en cours de montage, comportent des roues de $6900 \mathrm{~mm}$.

En dehors des soviétiques, les autres constructeurs étrangers ont construit plus de 200 groupes axiaux de plus de $1000 \mathrm{~kW}$ de puissance unitaire. Les diamètres des roues de ces machines axiales restent inférieurs à $5 \mathrm{~m}$, sauf celles prévues pour les installations de Ottensheim (sur le Danube), Iffezheim (sur le Rhin) et Hedmark en Norvège, dont les diamètres a'tteignent $5600 \mathrm{~mm}$.

Les deux constructeurs soviétiques de Leningrad et de Kharkov ont construit 56 groupes bulbes; pour 54 de ces machines les diamètres de roue vont de 5,5 à $6 \mathrm{~m}$ et les puissances de 18 à $23 \mathrm{MW}$. Les deux groupes expérimentaux de Saratov comportent des roues de $7,5 \mathrm{~m}$ de diamètre et développent $47,3 \mathrm{MW}$ sous $15 \mathrm{~m}$ de chute.

Maîtres d'œuvre et constructeurs français, promoteurs de cette technique, voient avec grand contentement l'accomplissement de ce qu'ils attendaient. Les constructeurs se doivent de remercier les clients français pour leur soutien enthousiaste qui a stimulé le développement de ces machines. Leur attitude constructive a grandement favorisé la résolution des quelques difficultés inévitables, apparues lors de la mise en exploitation des premiers groupes bulbes de grandes dimensions. 
Après le passé, examinons le proche avenir.

Le tableau ci-après donne les caractéristiques des principaux projets en étude:

\begin{tabular}{|c|c|c|c|c|}
\hline NOM DU SITE & PAYS & $\begin{array}{c}\text { NOMbRE } \\
\text { DE } \\
\text { GROUPES }\end{array}$ & $\begin{array}{c}\text { Puissance } \\
\text { (MW) }\end{array}$ & $\begin{array}{l}\mathrm{H} \\
(\mathrm{m})\end{array}$ \\
\hline $\begin{array}{l}\text { Péage- } \\
\text { de-Roussillon }\end{array}$ & France & 4 & 40 & 11,40 \\
\hline Belver & Portugal. & 1 & 34 & 12 \\
\hline Crestuma & Portugal. & 3 & 35 & 10,50 \\
\hline Rock-Island & U.S.A. & 8 & 56 & 13,30 \\
\hline Racine & U.S.A. & 2 & 19 & 6,70 \\
\hline Vancebourg & U.S.A. & 3 & 25 & 8,40 \\
\hline Canelton & U.S.A. & 3 & 25 & 8,40 \\
\hline Portes de fer & $\begin{array}{l}\text { Roumanie } \\
\text { Yougoslavie }\end{array}$ & 16 & 28 & 7,45 \\
\hline \multirow{2}{*}{$\begin{array}{l}\text { Chocon } \\
\text { Aval }\end{array}$} & \multirow{2}{*}{ Argentine } & 3 & 25 & 10 \\
\hline & & 3 & 38 & 15 \\
\hline
\end{tabular}

Certains de ces projets, en cours d'étude, comporteraient des machines avec des roues de $7500 \mathrm{~mm}$ de diamètre, cet accroissement des dimensions soulève de nouveaux problèmes. Signalons tout d'abord que la facilité de montage de l'alternateur, qu'offre la disposition avec chambre d'eau, rend, pour les groupes de grandes dimensions, cette solution beaucoup plus favorable que celle « en conduite ». La chambre d'eau permet aussi de monter séparément la turbine et l'alternateur et de gagner ainsi en délai; de plus, les supports «amont», situés au droit de l'alternateur, ont moins d'influence sur les écoulements et peuvent donc être mieux adaptés à leur fonction. Un demi-avant distributeur à la partie supérieure, disposition mécanique adoptée pour les groupes de Beaucaire, Gervans, Avignon, complèterait les appuis du groupe. Le bon fonctionnement de ces machines de grandes dimensions exige que les déformations des supports restent faibles par rapport aux déplacements relatifs admissibles des parties tournantes et des parties fixes. Le calcul de ces déformations doit tenir compte de tous les régimes de fonctionnement ainsi que des inégalités d'échauffement.

L'augmentation des dimensions exige une étude plus approfondie du dimensionnement des parties tournantes et de leurs appuis. $I$ faut contrôler sérieusement que forces et couples pulsatoires, d'origine hydraulique ou électromagnétique, nexcitent pas les premiers modes de vibrations (torsion, flexion) des parties tournantes. La disposition avec trois paliers s'impose pratiquement pour les groupes bulbes de grandes dimensions. Le calcul des réactions sur les paliers, pendant les régimes transitoires et à l'emballement, doit prendre en compte les efforts et couples pulsatoires 'd'origine hydraulique. Pour des groupes développant 40 à $50 \mathrm{MW}$, et comportant des roues de $7,5 \mathrm{~m}$ de diamètre, la charge sur le palier turbine dépasse $250 \mathrm{t}$. Deux des groupes qui équipent la centrale de Gerstheim, sur le Rhin, comportent des paliers à patins. Le palier, côté turbine, dont la charge peut atteindre $130 \mathrm{t}$, comprend quatre patins, seuls les deux situés à la partie inférieure, de part et d'autre du plan vertical médian, supportent la charge. Les deux patins logés à la partie supérieure, limitent les déplacements de l'arbre et ne travaillent que lorsque les efforts hydrauliques soulèvent les parties tournantes pendant certains régimes transitoires. Le deuxième palier, placé à l'amont de l'alternateur comporte cinq patins dont trois à la partie inférieure. L'axe de pivotement du patin le plus bas se situe dans le plan médian du palier, ce patin supporte donc la majeure partie de la charge. Pour des charges de 100 à $130 \mathrm{t}$, on pourrait aussi prévoir un seul patin inférieur d'angle au centre voisin de $90^{\circ}$. Avec des charges de l'ordre de $250 \mathrm{t}$, on a intérêt à réduire la surface des patins pour limiter les déformations d'origine mécanique et thermique; l'adoption d'une double couronne de patins constitue alors une solution satisfaisante. Une bonne répartition des charges entre les patins, et le maintien de la position relative des patins sous charge, restent les conditions indispensables à satisfaire. Précisons, pour conclure, que les essais effectués sur les paliers à patins des machines de Gerstheim, avaient été précédés d'une étude théorique approfondie et 'd'essais sur un modèle réduit [20].

L'énorme volume de mesures sur machines industrielles concernant les forces hydrodynamiques perturbatrices, le comportement mécanique d'ensemble ainsi que les nombreux essais sur modèle réduits hydrauliques et mécaniques, nous permettent d'envisager avec une absolue confiance la construction de groupes bulbes munis de roues dépassant $7,5 \mathrm{~m}$ de diamètre et capables de développer des puissances de plus de $50 \mathrm{MW}$. Ces machines viendront compléter la gamme des 212 groupes bulbes, dont 74 dépassent $5 \mathrm{~m}$ de diamètre, 26 , $6 \mathrm{~m}$, et 4 atteignent $6,9 \mathrm{~m}$ : ensemble de machines construites par les constructeurs français du groupe N-CL.

\section{Bibliographie}

[1] G. Martin et H. Giraud. - La séparation des pertes dans unc turbine Kaplan et une turbine axiale. A.I.R.H., Symposium de Nice, septembre 1960.

[2] P. Cazenave. - Un exemple d'aménagement de basse chute équipé de groupes bulbes: Pierre-Bénite sur le Rhône. S.F.E., Congrès de Brest, septembre 1963.

[3] S. Casacci, G. Ruelle, H. de Vergnette, - Economie des réalisations actuelles des groupes bulbes de grande puissance et perspectives d'avenir. Conférence Mondiale de l'Energie, Lausanne, septembre 1964.

[4] L. ERemeef. - Ecoulement à travers les grilles rectilignes en fluide parfait incompressible. Rapport interne Alsthom-Neyrpic, 1969-1972.

[5] L. ERemeef et Vinh-Phong. - Ecoulement à travers des grilles d'aubes tracées sur une surface de révolution. Rapport interne Alsthom-Neyrpic, 1969-1972.

[6] L. EREMEeF. - Ecoulement à travers des grilles d'aubes tracées sur une surface de révolution. Résolution de l'équation intégrale. Rapport interne Alsthom-Neyrpic, 1969-1972.

[7] E. BAER, - Mesure du champ d'écoulement relatif de l'eau sur les aubes d'une turbine Francis rapide. Forsch Ing. Wes., $36, \mathrm{n}^{\circ} 2,54-65,1970$.

[8] A. Ja. Aronson, - Evaluation de l'effet d'une erreur d'exécution d'une aube sur le coefficient de cavitation. Energomachinostroenie, $\mathrm{n}^{\circ} 8,1966$.

[9] S. CASACCI. - Advances in Low-head Machines Water Power, February, March, April 1961.

[10] S. Casacci, P. Jarriand, - Efforts de manouvie des organes de vannage des turbo-machines axiales en régimes permanent et transitoire. A.I.R.H., Symposium de Nice, 1960. 
[11] L. Kammerlocher. - Groupes générateurs hydroélectriques immergés type bulbe. Développement et évolution constructive. R.G.E., 1957 .

[12] J. Duclos. - Projets d'usines marémotrices. S.F.E., 1958.

[13] L. Kammerlocher. - La station marémotrice expérimentale de Saint-Malo. R.G.E., t. 69, p. 237-264, mai 1960.

[14] S. Casacci, L. Kammerlocher. - Analyse du fonctionnement des groupes axiaux de grandes dimensions. La Houlle Blanche, $\mathrm{n}^{0}$ spécial « Rance », 2/1962.

[15] P. Delattre, M. Henry, P. Cazenave. - Progrès techniques et économiques résultant de l'emploi des groupes axiaux dans les usines de basse chute, ou à titre d'équipement accessoire dans les barrages de ces mêmes usines, ou dans les digues des bassins de réfrigération des grandes centrales thermiques. Union internationale des producteurs et distri- buteurs d'énergie électrique. Congrès de Scandinavic, 23-30 juin 1964.

[16] La Rance: Une grande réalisation inédite. Revue française de l'énergie, numéro spécial, sept.-oct. 1966.

[17] M. L. STEKLo. - Groupes bulbes expérimentaux pour la centrale de Saratov. Energomachinostroenie, n ${ }^{\circ}$ 5, 1969.

[18] W. RosenmanN. - Experimental Investigations of Hydrodynamically Induced shaft Forces with a three bladed Inducer Cavitation in fluid Machinery. Winter annual meeting A.S.M.E., Chicago, November 1965.

[19] J. LHermitte. - Analyse des contraintes relevées sur une pale du groupe A3N de la Rance. La Houille Blanche, $\mathrm{n}^{\circ}$ 5, 1968.

[20] G. PÈRE. - Etude d'un palier porteur à patins pour turbines hydrauliques à axe horizontal. Mémoire CNAM, 1966.

\section{Discussion}

Président: M. P. CAzENAve

M. le Président remercie chaleureusement $M$. Casacci de son très intéressant exposé et ouvre la discussion:

M. KAMMERLOCHER demande quelles sont les limites actuelles des groupes bulbes en ce qui concerne leur fonctionnement en pompe (hauteur de refoulement limite, en particulier)?

M. CASACCr, d'accord avec M. DUPORT, n'estime pas raisonnable de faire fonctionner de telles machines sous des hauteurs de refoulement supérieures à $20 \mathrm{~m}$. Si cette valeur n'est pas un butoir du point de vue technique, elle l'est très probablement du point de vue économique, notamment en ce qui concerne l'enfoncement nécessaire pour limiter les usures dues à la cavitation.

M. ANDRÉ intervient en ces termes:

M. CASACCI, dans son exposé, a fait allusion à des solutions d'avenir concernant d'une part les accès à la turbine par l'alternateur, ct d'autre part un cercle de vannage situé à l'intérieur du conduit.

Les solutions envisagées attirent de la part de l'exploitant les deux remarques suivantes:

$1^{\circ}$ Il est vivement souhaitable de pouvoir accéder à la turbine sans avoir à passer par l'alternateur. En effet, plusieurs incidents sembient consécutifs à des blessures d'isolant causées par le passage des hommes à l'intérieur du stator. Dans le cas où il est impossible de faire autrement, il est recommandé de prévoir des caches permettant la circulation sans endommagement de l'isolant.

$2^{\circ}$ Dans le cas où le cercle de vannage ne serait pas accessible, il paraît fondamental que les pièces de rupture (biellettes de rupture ou goujons de cisalllement) le soient aisément, car il semble que le nombre de ruptures constatées sur les bulbes soit un peu plus important que dans les Kaplan. Ceci n'est pas une certitude et tient peutêtre seulement au fait que le nombre de machines par usine est généralement plus grand pour les bulbes, aucune statistique précise n'ayant été établie à ce sujet. Toutefois, les vitesses spécifiques élevées, l'espacement des grilles et le type d'écoulement rendent vraisemblable le passage d'un nombre plus grand de corps entre deux eaux dans les directrices.

M. CASACCI répond en ces termes:

$1^{\circ}$ La question se pose de savoir si avec des machines encore plus fiables que les groupes bulbes actuels, il restera rentable de prévoir des puits d'accès qui augmentent la longueur des groupes et provoquent des sillages dans le distributeur. Le constructeur se pliera cependant aux désirs de l'exploitant qui seul, peut décider en définitive.

$2^{\circ}$ Lorsque la commande de vannage sera située à l'intérieur du bulbe, il sera bien entendu indispensable que tous les mécanismes de cette commande soient accessibles. On peut prévoir un passage à travers l'alternateur ou par une cheminée verticale prévue dans une avant-directrice.

M. MEYsTre ne croit pas que l'aménagement d'un puits d'accès aux paliers côté turbine diminue le rendement de la turbine bulbe. Escher Wyss dut procéder à des essais comparatifs tres poussés pout les groupes de Treves (Moselle) et constata que, si les puits daccès et le socle du bulbe sont bien carénés et les sections d'écoulcment bien choisies, il n'y a pas de perte de rendement mesurable (voir p. ex. Bulletin Escher Wyss, 1957, 2\%). La proposition de supprimer cet accès ne peut être justifiée, à son point de vue, que par un désir d'économiser sur la construction au détriment de l'exploitation. Car le personnel a certainement le plus grand intérêt à pouvoir accéder directement et en service aux parties délicates que sont le palier, la butée et le joint d'arbre.

Mais, répond $M$. Duport, le rendement de la turbine n'est pas la seule considération qui intervient dans la détermination des moyens d'accès. Dans les machines très poussées, la présence d'un sillage ou même simplement d'un défaut par rapport à l'axi-symétrie de l'écoulement à l'amont du rotor peut être à l'origine, non seulement de pertes de rendement — et je reconnais qu'elles peuvent être extrêmement modestes - mais de l'apparition de cavitation, à l'origine de vibrations et de fatigue. Construisant des machines que l'on voulait très poussées, pour les raisons économiques que $M$. CASACCI a fort bien exposées, il est évident que nous avons été extrêmement circonspects quant à la présence de ces sillages.

Je crois que l'attitude à observer sur ce point dépend beaucoup du caractère plus ou moins poussé du rotor du point de vue cavitation, fatigue et risques éventuels de vibrations des aubages.

M. Meystre ouvre un débat sur un vieux mais fort intéressant problème par l'intervention ci-après :

Je suis heureux, dit-il, que M. CASACCI ait montré un diagramme comparatif des pertes dans un aspirateur coudé (cas des Kaplan) et un aspirateur rectiligne (cas des groupes bulbes). D'autre part, M. ANDRé, de son côté, a signalé que les performances de l'aspirateur des groupes bulbes ne s'étaient pas révélées aussi nettement supérieures à celles des aspirateurs coudés que l'on espérait à l'origine.

C'est lì un point très important sur lequel j'aimerais revenir. Il faut rappeler que l'influence de la forme de l'aspirateur - condé ou rectiligne - n'est pas du tout négligeable, contrairement à ce qui vient d'être dit. On constate d'abord que la part de transformation d'énergie dans la turbine à attribuer au diffuseur augmente considérablement avec la vitesse spécifique du groupe et atteint 40 à $45 \%$ pour un $n_{s}$ de 900 . Une variation de $10 \%$ du rendement de l'aspirateur y entraine donc une variation de 4 à $5 \%$ du rendement de la turbine. Or les pertes dans l'aspirateur d'une turbine rapide représentent plus de la moitié des pertes totales à pleine charge.

Que la transformation d'énergie cinétique en énergie de pression s'effectuera avec un meilleur rendement si elle n'est pas accompagnée d'un changement de direction est évident. En outre, un tourbillon - qui existe à l'cntrée de l'aspirateur pour toute charge non optimale - est ralenti et disparaît presque dans un diffuseur droit et circulaire tandis qu'il persiste, horizontal, jusqu'en aval de la sortie d'un aspirateur coudé, occasionnant parfois même, d'un côté, un retour de courant dans le diffuseur, ce qui détruit l'effet de décélération que lon en attend. La plus grande partie de la différence de rendement entre les deux types de turbines revient donc certainement at laspiraten rectiligne plus favorable. 
D'allleurs, des essais comparatifs, effectués sur un même modèle de turbine Kaplan avec bâche spirale, montrèrent une amélioration analogue du rendement à pleine charge par le simple remplacement d'un aspirateur coudé par un diffuseur droit. Il faut donc admettre, les pertes d'énergie dans la bâche spirale étant faibles, que l'amélioration du rendement par suppression de la spirale serait presque insignifiante (au maximum de $1 \%$ à pleine charge).

Ceci souligne bien l'influence du diffuseur sur le rendement et celle de la forme de l'entrée sur le débit maximal ou spécifique. Les deux parlent en faveur de la turbine bulbe. Et M. Meystre pense que ces qualités ont probablement autant de poids que l'économie que ces turbines permettent sur le génie civil, surtout lorsqu'on voit la rapidité avec laquelle l'argent se dévalue tandis que l'énergie hydraulique devient toujours plus précieuse.

Je ne pense pas qu'il y ait divergence de vue entre M. André et M. Merstre sur ce point, répond M. Casacci. Dans les mesure faites sur nos groupes bulbes. M. ANDRÉ précise qu'il n'a pas trouvé des rendements d'aspirateurs supérieurs à ceux obtenus sur des Kaplan. J'ai signalé que nous ne recherchions pas au début de nos études sur les machines axiales un accroissement des rendements, mais une augmentation des puissances spécifiques. En ce qui concerne l'influence des accès sur les performances de la machine, il va de soi qu'elle dépend beaucoup de la puissance spécifique.

M. Meystre ne parait pas d'accord.

M. Dourlete (E.D.F., D.T.G.) précise que les mesures rapportées par M. ANDRÉ ont simplement montré que les rendements d'aspirateur de Kaplan, obtenus à partir de mesures au tube de Pitot sous les roues, sont parfois aussi bons que ceux qui ont pu etre mesurés sur des groupes bulbes, mais il est hors question de conclure que les aspirateurs de groupes bulbes ne sont pas en moyenne meilleurs quo les aspirateurs coudés des machines verticales.

M. Meystre estime que comparer des rendements d'aspirateurs sur place est une impossibilité physique, parce qu'un tube de Pitot introduit dans l'écoulement existant à l'aval d'une roue ne renseigne que très mal sur l'énergie totale cle l'eau dans ladite section. Or, le rendement en question est basé sur la transformation de l'énergie entre le début et la fin de l'aspirateur. Si l'on veut comparer des aspirateurs de deux types, il faut que l'écoulement qui les précède soit analogue sous tous rapports.

M. Douillet conteste ce point de vue en se référant à de nombreuses mesures au tube de Pitot sous les roues Francis et Kaplan; un mode de dépouillement judicieux de ces mesures conduit à des résultats en bonne concordance avec ceux obtenus sur modèle réduit.

M. Duport apporte au débat - qui reste ouvert sur ce point la contribution suivante

On peut évidemment considérer le rendement de laspirateur, comme le disait $M$. Meystre à l'instant, en prenant en compte la différence des niveaux d'énergie à l'entrée et à la sortie de l'aspirateur. Il est apparu, au cours d'études qui ont été largement exposées et discutées à la Société Hydrotechnique de France, que l'énergic cinétique disponible à la sortic de l'aspirateur d'un groupe de basse chute - c'est particulièrement net pour les groupes axiaux - n'était pas totalement perdue et que selon le tracé des ouvrages aval, une partie plus ou moins importante de cette énergie pouvait être récupérée à l'aval de l'aspirateur.

Ce qui précède appelle de ma part les deux remarques suivantes:

a) Si l'on veut parler en pleine connaissance de cause, de comparaison d'aspirateurs, il est nécessaire, me semble-t-il, d'avoir effectivement mesuré les niveaux réels d'énergie à lentrée et à la sortie des aspirateurs, ce qui ne pent se faire à partir de la pression et de la vitesse moyenne, mais nécessite l'exploration des champs de vitesse et de pression.

b) Pour des raisons dapplication pratique, il est probablement utile de prendre en considération dans cette comparaison la récupération partielle qui se produit à l'aval de l'aspirateur. Je suggère, sur ce dernier point de se référer aux publications de la S.H.F. qui ont rendu compte des travaux poursuivis de facon concertée par la Direction des Recherches d'E.D.F. et par SOGREAH.

Du point de vue économique, quelle est la hauteur de chute limite des groupes bulbes fonctionnant en turbine, demande M. Cotillon?

Actuellement des groupes bulbes fonctionnent sous des chutes de $19 \mathrm{~m}$ répond $\mathrm{M}$. Casaccr. Au-dessous de 16 à $17 \mathrm{~m}$ de chute, les groupes bulbes se révèleront certainement plus économiques que les turbines Kaplan. Pour des chutes supérieures, le choix du type de machine dépendra beaucoup des caractéristiques spécifiques de I'installation.

Je pense, observe M. le President, que ces limites seront à revoit d'ici quelques années, lorsque les plus gros groupes, et notamment les groupes fixes que l'on envisage à l'heure actuelle, seront à leur tour considérés comme des groupes classiques.

M. le Président clôt la discussion et tire en ces termes la conclusion de la séance:

Nous devons des remerciements à la Société Hydrotechnique de France qui a préparé les exposés d'aujourd'hui dans des styles qui ont été, en général, très différents. Mais je crois qu'il faut remercier très sincèrement tous ceux qui, aujourd'hui, sont venus rapporter à la tribune ainsi que tous ceux qui, dans l'ombre, avaient travaillé pour permettre l'établissement de ces rapports, et aussi, bien entendu, ceux qui sont intervenus.

Cela dit, je pense que vous permettrez à un Président qui est un pell dans l'action et qui, sur une quantité de questions qui ont été évoquées ici, aurait souhaité pouvoir ajouter quelques précisions, de dire que nous espérons trouver, dans La Houlle Blanche, très prochainement, beaucoup de renseignements numériques, d'indications, de nuances qui n'ont pu être exprimés ici.

Je ferai ensuite, en guise de conclusion, deux petites remarques, l'une historique qui se rapproche d'ailleurs un peu d'une intervention de M. Duport, l'autre à caractère économique.

En ce qui concerne l'histoire, vous avez pu le voir, M. Cotillon qui s'était chargé de cela et de bien autre chose, ne s'est pas contenté de constater des faits; il a fait ouvre d'historien authentique en s'attachant à retrouver les causes et en cherchant à bâttir à travers les événements une construction logique, parfois même trop logique, et c'est peut-être ce qui a poussé $M$. DUPORT à intervenir, car le travail de l'historien permet de donner des événements des explications scientifiques dont les protagonistes, dans l'action, n'avaient souvent qu'une vague intuition. Il en était ainsi, je l'avais personnellement noté, et c'est ce qu'a dit M. DuporT, d'un point qui a passé très vite dans le rapport et que M. CorrLlon n'a pas présenté. Il s'agit du fameux rapport d'équivalence de 1,15 entre les diamètres de machines verticales et les diamètres de bulbes, qui ponvait donner I'impression d'avoir été senti ou connu très tôt alors qu'en réalité il n'était même peut-être pas encore perçu par tous aujourd'hui. Ceci explique d'ailleurs certaines divergences dans les comparaisons, au moins dans les comparaisons économiques.

Je crois qu'il faut noter que, surtout dans son enfance, la progression d'une technique, comme celle de toutes les activités humaines, doit autant à l'intuition, à la passion et à la foi qu'à la construction scientifique; l'histoire des bulbes, qui aurait d'ailleurs un organigramme extrémement compliqué - et c'est ce qui, dans votre exposé, mériterait d'être schématisé - l'histoire des bulbes a été fort compliquée avant la percée finale de Saint-Malo (car c'est en fait Saint-Malo qui a fait la percéc finale comme cela a été dit par un intervenant). On pourrait tracer un schéma de cette histoire avec des «points de branchement» qui pourraient porter les noms des pionniers.

Toute l'histoire des bulbes est jalonnée de prophètes parfois mystérieux, comme ARNo-FrScher, parfois très réalistes comme Gumbal puis Chamayou, Faral, de Verdelhan, Kammerlocher sans lesquels la confiance, à un moment, aurait fléchi, ce qui aurait entraîné un abandon prématuré. Même si leur action parait maintenant légèrement ou largement dépassée, il fallait que leur fût rendu cet hommage avant qu'on se contente d'examiner les problèmes concrets et actuels de ces machines qui, en effet, sont devenues classiques.

Ma deuxième remarque portera sur des problèmes d'économie Nous avons souligné tout à fait au début de l'exposé que les bulbes sont des.objets vraiment bizarres; j'avais, en particulier, noté leur physiologie et leur mécanique particulières. La remarque de M. Cornllon m'a appris qu'ils avaient aussi quelques particularités historiques assez curieuses, puisque, souvent, leur historien est en avance sur leur promoteur, et, en tout cas, le rapport de lhistorien est en avance sur celui du constructeur. Mais du point de vue éco nomique se pose encore un problème: tout le monde est d'accord sur le fait qu'il y a eu une très grosse économie (en gros, reprenons notre million de dollars pour le premier pas qui consistait à passer du groupe vertical au groupe bulbe «régulé», et en gros à peu près $70 \%$ de cette économie en passant du groupe réglable au groupe «fixe »).

Mais quelqu'un a posé la question : à qui cette économic profite-telle? M. André a déia donné une réponse partielle; c'était lui, en fait, qui pouvait être le plus inquiet; il dit que c'est parfait. Si bien que j'ai l'impression que, là aussi, on doit ajouter une loi économique particulière à cette technique qui est probablement une sorte de répartition hugolienne, en ce sens que, de cette économic, comme dit le poète, «chacun en a sa part et tous lont en enticr».

(Applandissements.) 